\title{
Trivium
}

Revue franco-allemande de sciences humaines et sociales - Deutsch-französische Zeitschrift für Geistesund Sozialwissenschaften

$14 \mid 2013$

Le Saint-Empire à l'époque moderne

\section{Das Alte Reich im Blick der deutschen und französischen Historiographie: Einleitung zum Themenschwerpunkt}

Falk Bretschneider et Guillaume Garner

\section{(2) OpenEdition}

Journals

Édition électronique

URL : http://journals.openedition.org/trivium/4508

DOI : 10.4000/trivium.4508

ISSN : 1963-1820

\section{Éditeur}

Les éditions de la Maison des sciences de l'Homme

\section{Référence électronique}

Falk Bretschneider und Guillaume Garner, « Das Alte Reich im Blick der deutschen und französischen Historiographie: Einleitung zum Themenschwerpunkt », Trivium [Online], 14 | 2013, online erschienen am 16 September 2013, abgerufen am 22 September 2020. URL : http://journals.openedition.org/ trivium/4508; DOI : https://doi.org/10.4000/trivium.4508

Ce document a été généré automatiquement le 22 septembre 2020.

\section{cc)}

Les contenus des la revue Trivium sont mis à disposition selon les termes de la Licence Creative Commons Attribution - Pas d'Utilisation Commerciale - Pas de Modification 4.0 International. 


\title{
Das Alte Reich im Blick der deutschen und französischen Historiographie: Einleitung zum Themenschwerpunkt
}

\author{
Falk Bretschneider et Guillaume Garner
}

\section{NOTE DE L'AUTEUR}

Die Autoren danken Christophe Duhamelle herzlich für seine Hinweise und Anregungen, die in den Themenschwerpunkt eingeflossen sind. Darüber hinaus gebührt Dank dem Institut français d'histoire en Allemagne (IFHA) in Frankfurt/M. und seinem Direktor, Pierre Monnet, für die institutionelle und fachliche Unterstützung bei der Vorbereitung dieser Ausgabe, die nicht zuletzt Ausdruck ist für eine in langen Jahren erprobte und intensive Kooperation zwischen den beteiligten Institutionen. Die Fondation Maison des sciences de l'homme und das IFHA arbeiten darüber hinaus bei weiteren Projekten zusammen, u. a. im deutsch-französischen Netzwerk »Saisir l'Europe - Europa als Herausforderung «, das auch weitere Partnerinstitutionen von Trivium (CIERA, Centre Marc Bloch, DHI Paris) einbezieht (http:// www.saisirleurope.eu).

1 Für die meisten Historikerinnen und Historiker in Frankreich gleicht das frühneuzeitliche Heilige Römische Reich Deutscher Nation einem Buch mit sieben Siegeln. Trotz erster Anfänge eines wechselseitigen Blicks auf die deutsch-französische Geschichte auch in der Frühen Neuzeit ${ }^{1}$, steht das Alte Reich bis heute weitgehend im historiographischen Schatten. An den französischen Universitäten wurde seine Geschichte lange Zeit kaum gelehrt und erforscht. Im Themenspektrum der Agrégation, die eine zentrale Rolle bei der Fixierung kanonischer Wissensbestände im französischen Schul- und Hochschulsystem spielt, tauchte es bislang als eigenständiger Gegenstand gar nicht auf. Dementsprechend fehlt es in Frankreich auch an soliden 
Einführungen - im Gegensatz etwa zum angelsächsischen Sprachraum, wo Studierende, Forscher und das interessierte Publikum seit Jahren die Auswahl zwischen mehreren Gesamtdarstellungen haben. Die derzeit aktuellste unter ihnen - Joachim Whaleys 2011 erschienene zweibändige Studie Germany and the Holy Roman Empire ${ }^{2}$ - gibt nicht nur einen profunden Überblick über die Geschichte des Alten Reichs in der Frühen Neuzeit, sondern ist darüber hinaus eine von der Kritik gelobte eigenständige Forschungsleistung, die auch in Deutschland interessiert wahrgenommen wird. In Frankreich suchte man ein solches Werk vergeblich. Wer dort nach einem Einstieg in die Reichsgeschichte fragt, wird notgedrungen auf ein 1976 erstmals erschienenes und zuletzt 1993 neu aufgelegtes Bändchen aus der populären Reihe "Que sais-je?» verwiesen. Man muss seinem Autor, Jean-François Noël, zwar Bewunderung dafür entgegen bringen, dass es ihm gelungen ist, auf 126 Seiten die wesentlichen Züge der Reichsentwicklung von der Krönung Kaiser Ottos I. im Jahr 962 bis zur Niederlegung der Reichskrone durch Franz II. 1806 weitgehend klar und präzise zusammenzufassen. Mehr als einen ersten Einblick in die vor allem institutionelle Geschichte des Alten Reiches aber kann sein Abriss nicht bieten. ${ }^{3}$

2 Vor diesem Hintergrund braucht es sicher keiner weiteren Begründung dafür, dem französischsprachigen Publikum mit dieser Nummer von Trivium einige zentrale Texte der deutschen Forschung zur Reichsgeschichte in Übersetzung zugänglich zu machen. Die ursprüngliche Idee zu diesem Themenschwerpunkt hatte vorgesehen, das übliche Format der Zeitschrift zu verlassen und eine Ausgabe vorzubereiten, die allein Beiträge deutscher Historikerinnen und Historiker enthalten hätte. Rechnung getragen werden sollte damit dem eklatanten Ungleichgewicht der Reichsforschung auf beiden Seiten des Rheins*. Entstanden wäre also ein Panorama aus Arbeiten, die den Stand einer deutschen Historiographie widerspiegeln, die sich seit nunmehr drei Jahrzehnten intensiv mit der Geschichte des Alten Reichs auseinandergesetzt und dabei eine eigene Unterdisziplin - die »Reichsgeschichte« - hervorgebracht hat. Rasch aber wurde den Herausgebern klar, dass eine solche Zusammenstellung eine unverzeihliche Leerstelle aufgewiesen hätte: Die oben geschilderte weitgehende Unkenntnis der Reichsgeschichte mag für die Mehrzahl der französischen Historikerinnen und Historiker gelten. Aber auch in Frankreich hat es in den letzten Jahren vereinzelt ein verstärktes Interesse für das Alte Reich gegeben, das nicht nur erwähnenswert ist, sondern aufgrund des besonderen Blickwinkels dieser Arbeiten auch eine wichtige Ergänzung zur deutschen Forschung darstellt, indem es dort vorhandene blinde Flecken erhellt. Die deutschen Beiträge wurden deshalb durch einige französische Texte ergänzt, so dass der Themenschwerpunkt nun einen in dieser Form bislang nicht vorhandenen Einblick in eine neue Reichsgeschichte bietet, die nicht mehr allein national verankert ist, sondern von miteinander verschränkten Perspektiven aus unterschiedlichen geschichtswissenschaftlichen Traditionen profitiert. ${ }^{4}$ Ziel dieser Einleitung ist es, diesen Kontext näher vorzustellen und die Auswahl der versammelten Texte zu erläutern.

\section{1. »Reichsgeschichte«: eine (kurze) Bilanz der Forschung in Deutschland}

3 In der deutschen Historiographie ist die Geschichte des Alten Reichs in den vergangenen Jahrzehnten von einem weithin verschmähten Randphänomen zu einem 
zentralen Gegenstand der Forschung geworden. ${ }^{5}$ Um sich diesen Umschwung zu verdeutlichen, reicht ein Blick in die sukzessiven Ausgaben des Gebhardt, dem klassischen Handbuch zur deutschen Geschichte: In der Neubearbeitung der 9. Auflage (Taschenbuchausgabe,1973-1980) tauchte in den 28 Kapitelüberschriften des Bandes zur Frühen Neuzeit gerade einmal das Wort »Reich« auf (und tatsächlich behandelt wurde dieses auf nicht mehr als einer Seite); zwölf Kapitel hingegen beschäftigten sich ausweislich der Überschriften mit Preußen oder Österreich. 2006, dreißig Jahre später, führt der den Jahren 1648-1763 gewidmete Band der 10. Auflage nun nicht nur explizit das Reich im Titel, zwei der insgesamt drei Abschnitte sind auch mit »Reichsgeschichte« überschrieben, und kein Kapitel befasst sich mehr ausschließlich mit Preußen oder Österreich. ${ }^{6}$

Natürlich handelt es sich hierbei nur um einen Indikator, der noch dazu nicht besonders zuverlässig ist (weite Teile der deutschsprachigen Frühneuzeitforschung kommen auch heute ohne jeden Reichsbezug aus). Gleichzeitig symbolisiert er einen fundamentalen Wandel: Bis in die Zeit nach dem 2. Weltkrieg hinein wurde dem Alten Reich von den meisten deutschen Historikern eine an Verachtung grenzende Geringschätzung entgegengebracht. Als wirkmächtig erwiesen sich dabei konfessionelle Prägungen (kurzgefasst galt das katholische Kaisertum der mehrheitlich protestantisch orientierten Geschichtswissenschaft schlicht als "undeutsch«), vor allem aber die für die Disziplin konstitutive Konzentration auf die als verspätet wahrgenommene deutsche Nationalstaatsbildung, die eben nicht an das Reich, sondern an den politischen und militärischen Aufstieg Preußens gekoppelt wurde. ${ }^{7}$ Seit den 1960er Jahren und im Zuge einer stärker werdenden kritischen Auseinandersetzung mit dem Nationalsozialismus erhielt das Reich jedoch - zumindest in der Bundesrepublik - neue Aufmerksamkeit als Teil einer deutschen Geschichte, die weder macht- noch nationalstaatlich geprägt war, sondern vermeintliche Anknüpfungspunkte bot für eine europäisch, föderalistisch, rechtsstaatlich, parlamentarisch und überkonfessionell ausgerichtete Tradition von Politik und Gesellschaft. ${ }^{8}$ Die in den Folgejahren einsetzende intensive Forschungstätigkeit ${ }^{9}$ - die ein wichtiges Forum auch in der 1974 gegründeten Zeitschrift für historische Forschung finden sollte - wandelte das Bild des Alten Reiches deshalb grundsätzlich und färbte es nun positiv, was zeitweise sogar zu einer regelrechten "Reichseuphorie« führte, die auch vor direkten Kontinuitätslinien etwa zwischen dem Regensburger Reichstag und dem Parlamentarismus der Bonner Republik nicht zurückschreckte. ${ }^{10}$

5 Tendenziell positiv gefärbt war ab der Mitte des 20. Jahrhunderts auch der Blick der deutschen Wirtschaftsgeschichte auf das Alte Reich. Bereits in den 1950er und 60er Jahren war das Reich in den Arbeiten von Wirtschaftshistorikern vielfach präsent - ein Interesse, das sich einerseits durch die Bedeutung des Staats in den Problemstellungen der allgemeinen Geschichte (»Staatsbildung ", »Staatswerdung«) und durch das Primat der Erforschung der Wirtschaftspolitik und der staatlichen Intervention erklärt, andererseits aber auch durch die bereits angesprochene "Reichsbegeisterung". Fruchtbar war diese Konjunktur vor allem für Studien zum Merkantilismus, die sich nicht allein auf die Territorialstaaten konzentrierten, sondern auch den sog. »Reichsmerkantilismus « einbezogen. ${ }^{11}$

6 Die auch hier z. T. spürbaren Auswüchse der Begeisterung und der Romantisierung des Reichs sowie die ihnen inhärente Tendenz zur Vereinnahmung mit dem Ziel der Befriedigung aktueller Orientierungsbedürfnisse werden inzwischen jedoch durchaus 
kritisch gesehen und hinterfragt. ${ }^{12}$ Auch können sie nicht verbergen, dass die Forschung bis heute mit grundsätzlichen methodologischen Schwierigkeiten $\mathrm{zu}$ kämpfen hat, die eine Beschäftigung mit der Geschichte des Alten Reichs keinesfalls zu einer historiographischen Selbstverständlichkeit werden lassen. Dazu gehören 1. die Definition des Gegenstandes »Reich« selbst, 2. der Maßstab, mit dem dieser Gegenstand wissenschaftlich erfasst werden kann, sowie 3. die zu seiner Erforschung adäquaten historiographischen Ansätze.

7 1.) Was ist das Reich?:

Wer über das Alte Reich arbeitet, arbeitet nicht einfach zum frühneuzeitlichen »Deutschland«. »Reichsgeschichte« zu betreiben heißt vielmehr, das Reich als eine zumindest politische Realität ernst zu nehmen. Worin bestand aber diese Realität? Diese Frage berührt einen neuralgischen Punkt der Reichsforschung, denn das Problem beschäftigt nicht erst die Historiker. Es bildet vielmehr den Kern einer Diskussion, die bereits die Zeitgenossen im 17. Jahrhundert führten und die zentral um die Frage kreist, mit welchen politischen und rechtlichen Kategorien sich dieses merkwürdige Verfassungsgebilde begreifen ließe. ${ }^{13}$ Seit dem Erscheinen der Six livres de la République von Jean Bodin (1576), die auch im deutschsprachigen Raum intensiv rezipiert und diskutiert wurden, geht die politische Theorie davon aus, dass staatliche Macht nur an eine einzige Quelle von Souveränität oder majestas gebunden sein könne. Im Reich aber fehlte diese eine souveräne und absolute Macht - in die Herrschaftsgewalt teilten sich hier Kaiser und Stände (symbolisiert insbesondere durch die herausgehobene Rolle der Kurfürsten, die den Kaiser wählten, gleichzeitig aber, wie alle anderen unmittelbaren Reichsglieder, ihre Territorien und Regalien von ihm zum Lehen erhielten ${ }^{14}$ ). Metaphorisch wurde das Reich von den Zeitgenossen deshalb oft als ein Körper aus Haupt (Kaiser) und Gliedern (Stände) beschrieben, der nur als Ganzes handlungsfähig war. ${ }^{15}$ Der Kaiser war zwar oberster Lehnsherr, oberster Richter, oberster Wahrer von Frieden und Recht. Ein absoluter Herrscher aber war er nicht. Vielmehr blieb er immer vom Konsens mit den anderen Reichsmitgliedern abhängig.

Diese Abhängigkeit vergrößerte sich sogar noch im Laufe der Frühen Neuzeit. Im Westfälischen Frieden von 1648 war es den Reichsständen gelungen, dem Kaiser eine ganze Reihe von Konzessionen abzuringen, die darauf hinausliefen, die Verbindungen zwischen Reichsoberhaupt und Reichsgliedern mehr und mehr nach den Regeln des neu entstandenen Völkerrechts zu gestalten. Tendenziell bedeutete dies, die alten, vom Prinzip persönlicher Abhängigkeit geprägten Lehnsverhältnisse durch ein neues Miteinander auf Augenhöhe abzulösen. Die politischen Beziehungen im Reich oszillierten in den folgenden anderthalb Jahrhunderten deshalb beständig zwischen der alten Idee des Lehnsverbandes und der neuen Vorstellung einer Föderation aus mehr oder weniger souveränen Staaten. ${ }^{16}$ Dabei bildete sich zunehmend ein Ungleichgewicht heraus zwischen den bedeutenderen Reichsständen (insbesondere den Kurfürsten, von denen im 18. Jahrhundert mehrere - Brandenburg, Sachsen, Hannover - auswärtige Königswürden erwarben) und den "mindermächtigen" Reichsgliedern (kleine und kleinste Fürsten- und Herzogtümer, Grafschaften etc., die angesichts der ständig lauernden Gefahr der Mediatisierung durch ihre mächtigeren Reichsnachbarn den Schutz des Kaisers suchten).

9 Verfassungsrechtliche Klarheit suchte man im Fall des Alten Reiches also vergeblich. ${ }^{17}$ Dazu trug auch bei, dass aufgrund zahlreicher ineinander laufender Herrschaftsrechte, die sich überlagerten, kreuzten oder sogar widersprachen, die Grenzen zwischen den 
Territorien der einzelnen Reichsstände nicht selten unklar und umstritten waren. ${ }^{18}$ Bei den Historiographen des 19. Jahrhunderts, denen die Legitimierung der nationalstaatlichen Modernität eine nahezu missionarische Aufgabe war, konnte das Reich deshalb nur negative Assoziationen hervorrufen. Für Heinrich von Treitschke, Kopf der borussisch-kleindeutschen Schule, befreite so erst der Aufstieg des protestantischen Preußen das Vaterland aus »dem Durcheinander verrotteter Reichsformen und unfertiger Territorien« und hauchte der "zerrissenen deutschen Nation" wieder "Lebenskraft" ein. ${ }^{19}$ Eine solche apodiktische Heftigkeit in der Verurteilung war zwar nicht die Regel, legte aber mit ihrer Sicht auf das Alte Reich als politische Abnormität, als pathologische Ausnahme im europäischen Staatsbildungsprozess und als entscheidendes Hemmnis für das Werden einer deutschen Nation den Grundstein für eine Perspektive, die in ihrer Gegenüberstellung von Reich und Nationalstaat bis in die aktuellen geschichtswissenschaftlichen Debatten nachwirkt. Gewissermaßen unter umgekehrten Vorzeichen fortgeführt wurde sie in der jüngsten Vergangenheit mit der Diskussion um ein 1999 erschienenes Buch des Jenenser Historikers Georg Schmidt, der vorschlug, das Reich als einen »komplementären Reichs-Staat« zu verstehen, der sich nicht grundsätzlich von anderen politischen Gebilden der europäischen Frühen Neuzeit unterschieden habe. ${ }^{20}$ Schmidts These und ihre Diskussion bilden den Gegenstand der beiden ersten Artikel dieses Themenschwerpunkts, weshalb wir später noch einmal auf sie zurückkommen werden.

10 2.) Wo ist das Reich zu finden?:

Es ist bereits weiter oben gesagt worden: Auch heute noch kommt ein Großteil der deutschen Forschung zur Frühen Neuzeit nahezu ohne jeden Bezug zum Reich aus. ${ }^{21}$ In der Historischen Kriminalitätsforschung etwa, einem besonders dynamischen Forschungszweig der letzten drei Jahrzehnte, ist es üblich, Arbeiten auf der Ebene einzelner Territorialstaaten oder Reichsstädte anzusiedeln. Das Reich hingegen wird nur äußerst selten in den Blick genommen. ${ }^{22}$ Gleiches gilt auch für die Politikgeschichte. Zwar wird hier immer wieder auf die Verbindungen zwischen einzelnen Territorien bzw. diesen und dem Reich eingegangen, die Präsenz des Reiches im politischen Funktionieren des jeweiligen Territoriums selbst jedoch bleibt weitgehend ausgeblendet. "Reichgeschichte« ist deshalb bis heute oftmals allein Gegenstand der Subdisziplin gleichen Namens, wobei dort die Aufmerksamkeit wiederum nahezu ausschließlich auf den Reichsinstitutionen (Kaiser, Reichstag, Reichsgerichte usw.) liegt. Erst langsam setzen sich Perspektiven durch, die übergreifende Praktiken thematisieren, etwa die Policeygesetzgebung, die im 16. Jahrhundert auf der Reichsebene initiiert wurde, bevor sie sich auf der Ebene der Territorien intensivierte ${ }^{23}$, oder die Reichskreise als Rahmen einer territorial übergreifenden Form der politischen Kooperation.

11 Feststellen lässt sich eine gewisse Marginalisierung des Reichs (nach einer anfänglichen Aufgeschlossenheit, siehe oben) seit den 1970er Jahren auch in der Wirtschaftsgeschichte. Merklich spürbar ist hier die Durchsetzung des Modernisierungsparadigmas bzw. die Dominanz von Arbeiten, die sich mit dem Übergang von einer Ökonomie des Ancien Régime $\mathrm{zu}$ ersten industriellen Wirtschaftsformen beschäftigen. Studien aus dem Umkreis der Historischen Sozialwissenschaft, aber auch Arbeiten zu den Gewerbelandschaften oder zur Protoindustrialisierung übergehen das Reich so völlig (Gleiches gilt auch für in der DDR entstandene wirtschaftshistorische Arbeiten). Hier wie in Untersuchungen zur 
Geschichte der ökonomischen Diskurse und Wissensbestände liegt die Aufmerksamkeit eindeutig auf der Ebene der Territorialstaaten, die als Ursprungsort des Kameralismus im 18. Jahrhundert angesehen werden. Vernachlässigt wird dabei der Umstand, dass auch das Reich Gegenstand ökonomischer Reflexion war $^{24}$ bzw. dass diese Diskurse innerhalb des Reichsraums zirkulierten. Exemplarisch für den Rückgang wirtschaftshistorischen Interesses für das Reich zitieren lässt sich paradoxerweise das bereits genannte Buch von Georg Schmidt, der zum Handeln des »komplementären Reichs-Staats « im Bereich der Ökonomie kaum ein Wort verliert. ${ }^{25}$

12 Kehren wir also zu der Frage zurück, auf welcher Ebene das Reich zu finden ist: Als Lehnsverband umfasste es zunächst alle Vasallen, die ihre Territorien und Regalien vom Kaiser zum Lehen erhielten (davon ausgenommen, gleichfalls aber Mitglieder des Reiches waren die Reichsstädte und Reichsdörfer, die keine Vasallen, sondern direkte Untertanen des Kaisers darstellten). In dieser Definition erstreckte sich das Reich bis weit nach Italien hinein und bezog auch Gebiete in den Niederlanden (bis 1548), in der Schweiz (bis 1648 und z. T. noch länger) oder im deutsch-französischen Grenzraum ein (Freigrafschaft Burgund, Bistümer Metz, Toul, Verdun, Reichsstadt Straßburg - diese Gebiete fielen im Zuge der französischen Expansionspolitik allerdings nach und nach an Frankreich). ${ }^{26}$ Einzelne Reichsterritorien unterstanden der Landesherrschaft auswärtiger Monarchen, die damit zugleich Reichsfürsten waren (Holstein etwa war im Besitz der dänischen Krone, und Vorpommern gehörte seit dem Westfälischen Frieden zu Schweden). Daneben gab es Gebiete, die zwar in Verbindung mit dem Reich standen, ihm jedoch formal nicht angehörten. Dazu zählten etwa die südlichen Provinzen der Niederlande (das heutige Belgien), die unter der Hoheit der Habsburger standen, Besitzungen deutscher Fürsten im Elsass, das Herzogtum Lothringen (das lange Zeit zwischen der Zugehörigkeit zu Frankreich, dem Reich und einem unabhängigen Status schwankte) oder Preußen, das bis 1657 polnisches Lehen war, bevor es dem brandenburgischen Kurfürst gelang, dort die Souveränität als Herzog zu erlangen und sich 1701 selbst zum »König in Preußen« zu erhöhen. Auch das Königreich Böhmen war ein eigener Fall: Der böhmische König war zwar seit dem Mittelalter Lehnsmann des Kaisers und gehörte zum Kreis der Kurfürsten. Als Lehen galt aber nur die Kurwürde, nicht das Territorium selbst (zu dem als Nebenländer auch Mähren, Schlesien und die beiden Lausitzen zählten), weshalb Böhmen bis ins 18. Jahrhundert hinein auch nicht an den Reichsinstitutionen partizipierte. Schließlich zählten die österreichischen Erbländer der Habsburger zwar lehnsrechtlich zum Reich, nahmen aber durch den Umstand, dass die Habsburger in der Frühen Neuzeit nahezu ununterbrochen den Kaiser stellten, eine Ausnahmestellung ein. Das hat u. a. dazu geführt, dass die Geschichte Österreichs bis heute weitgehend österreichischen Historikern überlassen bleibt. ${ }^{27}$

13 Eine weitere Möglichkeit, das Reich in den Blick zu nehmen, besteht darin, von den schon mehrfach angesprochenen Reichsinstitutionen auszugehen. Dazu gehörten der Reichstag, auf dem (von Ausnahmen - wie etwa den Angehörigen der Reichsritterschaft - abgesehen) im Prinzip alle Reichsvasallen Sitz und Stimme hatten, die Reichsgerichte (Reichshofrat und Reichskammergericht) sowie die im Zuge der Reichsreform im Jahr 1500 erstmals eingerichteten Reichskreise. In diesen drei Einrichtungen wurde das Reich besonders manifest, weshalb sie in den letzten Jahrzehnten auch im Zentrum der deutschen Forschung gestanden haben. Besonders gut untersucht ist die Geschichte des Reichstags, der zunächst in lockerer Folge und an unterschiedlichen Orten tagte, bevor er ab 1663 als »Immerwährender Reichstag« dauerhaft in Regensburg verblieb. Seine 
Sitzungsakten werden seit 1867 in einem groß angelegten Editionsprojekt aufbereitet und der Forschung zugänglich gemacht. ${ }^{28}$ In den letzten Jahrzehnten zunehmend intensiv untersucht wurde auch die Tätigkeit der beiden Reichsgerichte: das in Speyer bzw. ab 1689 in Wetzlar ansässige Reichskammergericht und der in Wien tagende Reichshofrat. Beide Gerichte stellten zum einen wichtige Instanzen der Konfliktregelung zwischen Kaiser und Ständen bzw. zwischen einzelnen Territorien dar, zum anderen wurden sie immer wieder auch von Untertanen genutzt, die sich gegen obrigkeitliches Unrecht zur Wehr setzten. ${ }^{29}$ Als wichtiges Element der Reichsverfassung hat schließlich die Einteilung des Reichs in Kreise Aufmerksamkeit auf sich gezogen. Als überterritoriale Zusammenschlüsse waren diese u. a. für die Vollstreckung von Urteilen des Reichskammergerichts, für das Münzwesen oder die Kontingente der Reichsarmee zuständig. Besonders intensiv war die Zusammenarbeit dabei in Süd- und Südwestdeutschland (dort also, wo die politisch-geographischen Verhältnisse besonders zersplittert waren), während die Kreise im von größeren Territorialstaaten geprägten Osten und Norden zunehmend ihre Funktionen verloren. ${ }^{30}$

Bereits diese wenigen Einblicke machen deutlich, dass es keinen einheitlichen Maßstab gibt, mit dem sich das Reich erfassen ließe. Dabei spielt auch eine Rolle, dass sich mit diesem bis heute keine eigene archivalische Überlieferung verbindet. Vielmehr sind die Bestände der wichtigsten Einrichtungen des Reiches in der ersten Hälfte des 19. Jahrhunderts auf die neu entstandenen Staaten des Deutschen Bundes aufgeteilt worden. ${ }^{31}$ Quellenmäßig existiert das Reich deshalb nur auf dem Umweg über die Archive seiner Glieder - was wiederum die Frage aufwirft, ob es nicht auch außerhalb seiner selbst, $d$. h. außerhalb seiner zentralen Institutionen in Wien, Regensburg oder Wetzlar, eine Wirklichkeit hatte. In den Blick gerät der Reichsverband hier nicht als ein institutionell fixierter, sondern als ein relational gestifteter Zusammenhang. Ein erstes hervorstechendes Beispiel für eine solche relationale Reichsgeschichte ist die Reichspost, die 1495 von Kaiser Maximilian I. eingerichtet und 1615 der Familie Thurn und Taxis als erbliches Reichslehen übertragen wurde. ${ }^{32}$ Die Post verband mit ihrem regelmäßigen Brief- und ab dem späten 17. Jahrhundert auch Personenverkehr nicht nur die unzähligen Territorien des Reichs miteinander, über ihre mit dem doppelköpfigen Reichsadler versehenen Schilder hielt sie das Reich auch dort präsent, wo es durch die Dominanz größerer Territorialstaaten ansonsten nur noch wenig spürbar war. Ein zweites Beispiel sind die mit kaiserlichen Privilegien in Leipzig und in Frankfurt/M. abgehaltenen großen Messen, die unter dem besonderen Schutz des Reichsrechts nicht nur den Handel organisierten, sondern in der Form des freien Geleits den Händlern auch die dafür nötige Bewegungsfreiheit garantierten. ${ }^{33}$ Und drittens sind die Universitäten $\mathrm{zu}$ erwähnen, deren Lehrbetrieb ebenfalls auf kaiserlichen Privilegien beruhte und die genau aus diesem Grund in der Lage waren, sowohl Professoren als auch Studenten aus allen Gegenden des Reiches anzuziehen und Titel zu verleihen, die wiederum im gesamten Reich anerkannt wurden. ${ }^{34}$

Auch »im Reich" hatte das Reich also Orte. Das gilt ebenfalls für die Territorien. Gleichwohl steht die Forschung hier noch weitgehend am Anfang. ${ }^{35}$ Einzelne Arbeiten zeigen jedoch mögliche Richtungen auf, in denen sich eine solche Präsenz des Reichs auf der Ebene der Territorien erforschen ließe: Neben zahlreichen Publikationen zur »Reichspolitik« einzelner Reichsfürsten ${ }^{36}$ intensiver untersucht wurde so die Rolle der Mainzer Kurfürsten, die nicht nur Landesherren in ihrem eigenen Territorium, sondern auch Erzkanzler des Reiches waren und somit eine herausgehobene Rolle im institutionellen Gefüge des gesamten Reichsverbandes spielten. ${ }^{37}$ Andere Studien 
beschäftigen sich mit den Beziehungen einzelner Territorien zu den Reichsgerichten, etwa im Fall der Grafschaft Lippe, wo nicht nur die Obrigkeit, sondern auch die Untertanen derart häufig um die Hilfe von Reichshofrat und Reichskammergericht ansuchten, dass sich der regierende Graf gezwungen sah, bei jedem der beiden Gerichte einen ständigen Vertreter $\mathrm{zu}$ unterhalten. ${ }^{38}$ Schließlich wurde vereinzelt die Zwangsverwaltung untersucht, die besonders im 18. Jahrhundert zahlreiche (vor allem kleine und kleinste) Territorien und Reichsstädte traf, die ihre Schulden nicht mehr begleichen konnten. Diese durch die Reichsgerichte angeordnete und von sog. »Reichskommissionen« durchgeführte Maßnahme bedeutete für den betroffenen Fürsten oder Grafen zwar den nahezu kompletten Verlust seiner Autonomie, verhinderte gleichzeitig aber, dass sein Herrschaftsgebiet von den als Gläubigern auftretenden Nachbarterritorien mediatisiert wurde..$^{39}$

Dies sind einige Beispiele für die erst langsame Auflösung einer dichotomischen Perspektive, wie sie in den letzten Jahrzehnten auch die Reichsgeschichte mit ihrer Konzentration auf die zentralen Reichsinstitutionen nicht hat aufheben können. Auch hier standen sich Reich und Territorien vielfach noch als Antipoden gegenüber, die vor allem gegeneinander und kaum miteinander agierten. ${ }^{40}$ Erst neueren Ansätzen, welche die bislang vorherrschenden verfassungs-, institutionen- und im traditionellen Sinn politikgeschichtlich geprägten Orientierungen aufgebrochen und das Reich somit auch zur allgemeinen Geschichte hin geöffnet haben, ist es gelungen, in dieser Hinsicht Neuland zu betreten.

17 3.) Neue Ansätze der Reichsgeschichte:

Dazu gehört in erster Linie eine neue Kulturgeschichte des Politischen, der es u. a. darum geht, das Reich von der Last nachträglicher Bewertungen zu befreien und die Handlungslogiken der zeitgenössischen Akteure wieder stärker in den Mittelpunkt der Analyse zu stellen. Bislang dominante Fragen nach dem politischen "Charakter" des Reichs (Staat, Nation usw.) treten dabei zurück zugunsten einer Interpretation, die das in der politischen Praxis der verschiedenen Reichsglieder zutage tretende komplexe Miteinander ernst nimmt. Verwirklicht wurde dieser Ansatz vor allem in Barbara Stollberg-Rilingers Buch Des Kaisers alte Kleider, das anhand der Untersuchung von symbolisch-rituellen Formen (Thronbelehnungen, Kaiserkrönungen, Sitzungen des Reichstags usw.) einen grundsätzlichen Wandel in den Strukturen der politischen Kommunikation beschreibt: Die traditionelle Ständekultur beruhte auf einer hierarchischen Grammatik, deren Rituale die Lehnsabhängigkeiten ausdrückten, welche die Akteure miteinander verbanden. Ihr zur Seite trat im Laufe der Frühen Neuzeit eine neue, egalitäre Grammatik, deren Regeln sich nach den Gepflogenheiten des diplomatischen Verkehrs richteten, der bestimmte, dass sich souveräne Potentaten untereinander gleich behandelten. Im Fall des Alten Reichs blieb dieser Wandel jedoch unabgeschlossen. Auch hier strebten gerade die großen Fürsten seit dem ausgehenden 17. Jahrhundert nach dem Glanz ihrer eigenen Majestät. Gleichzeitig blieben sie aber Glieder in einem Lehensverband, der die wichtigste Basis ihrer Herrschaftsgewalt war. Resultat dieses Widerspruchs war eine »strukturelle Doppelbödigkeit« im Handeln der Akteure, die versuchten, im Rahmen einer »organisierten Heuchelei« beiden sozialen Logiken zu genügen, und deshalb in den Augen kritischer Zeitgenossen nur noch ein inhaltsleeres Schauspiel aufführten. ${ }^{41}$

18 Eine weitere Neuerung besteht darin, das Reich als einen übergreifenden Kommunikationszusammenhang in den Blick zu nehmen. Neben den bereits 
angesprochenen Forschungen von Wolfgang Behringer zur Geschichte der Reichspost (siehe oben Anm. 32) steht exemplarisch für einen solchen Ansatz Susanne Friedrichs Untersuchung des Regensburger Reichstags als Informations- und Kommunikationsknotenpunkt. Auch hier geht es darum, sich von alten Deutungen zu verabschieden, die die Vorgänge auf den zentralen Bühnen des Reichs im 18. Jahrhundert nur noch als institutionalisierten Mummenschanz begreifen, dessen Fortdauern sich kaum erklären lässt. Zwar widerspricht Friedrich nicht der Einschätzung, der Reichstag sei als politisches Verhandlungsforum um 1700 nur noch selten erfolgreich gewesen. Das aber, so ihre These, war auch gar nicht seine Funktion. Vielmehr stellte er gerade für mindermächtige Reichsstände, die sich kein aufwändiges Gesandtschaftswesen leisten konnten, eine eminent wichtige Informations- und Kommunikationsbörse dar. Hier erfuhren sie Neuigkeiten aus der politischen oder höfischen Welt (wobei bei der Informationsbeschaffung auch vor unorthodoxen Mitteln wie Bestechung, Verrat und dem Einsatz von Spionen nicht Halt gemacht wurde), hier tauschten sie sich mit den Repräsentanten anderer Mächte aus (in Regensburg waren auch zahlreiche Vertreter ausländischer Höfe akkreditiert) und von hier aus entspann sich ein das gesamte Reich umfassendes Kommunikationsnetz, das eine zentrale Grundlage für das Handeln der Reichsmitglieder bildete. ${ }^{42}$

Auch im Bereich der Wirtschaftsgeschichte lässt sich in letzter Zeit ein neuerwachtes Interesse für das Reich beobachten. Drei Tendenzen sind dabei erkennbar: Zum einen interessiert sich die Forschung für das wirtschaftliche Handeln der Reichsinstitutionen selbst. Hier handelt es sich vor allem um die Reichsgerichte und insbesondere das Reichskammergericht, dessen Rechtsprechung eine Bedeutung für das Wirtschaftsleben hatte, die zunehmend neu bewertet wird (die Arbeiten von Anette Baumann und - neuer noch - von Anja Amend-Traut sind in dieser Hinsicht vorbildhaft ${ }^{43}$ ). Hinsichtlich des Reichstags fällt der Befund hingegen weniger eindeutig aus: Zwar wurden seine Bemühungen um eine Vereinheitlichung des Münzwesens von der Geschichtswissenschaft immer wieder unterstrichen, die tatsächliche Reichweite seiner Entscheidungen bleibt jedoch noch zu untersuchen. ${ }^{44}$ Der Erfolg der Reichshandwerksordnung von 1731, die sich der Reform der Zunftverfassung angenommen hatte, wurde so lange Zeit bezweifelt. Aktuelle Arbeiten stellen im Gegenteil jedoch heraus, wie stark sie die Handlungsspielräume der territorialstaatlichen Regierungen in diesem Bereich erweiterte. ${ }^{45}$ Bemerkbar macht sich hier eine Schwierigkeit, die der Struktur des Reichs selbst inhärent war: legislative und exekutive Gewalt »teilten" sich Instanzen, die auf unterschiedlichen Ebenen agierten. Zum anderen ist die Forschung hinsichtlich der Reichskreise in den letzten Jahren ebenfalls $\mathrm{zu}$ neuen Ergebnissen gekommen. Das gilt insbesondere für drei Bereiche - das Straßennetz, den Getreidehandel und das Münzwesen ${ }^{46}$-, für welche die bisherige Literatur angenommen hatte, die territoriale Zersplitterung des Reichs stelle ein unüberwindliches Hindernis dar, das die Akteure zu weitgehender Untätigkeit verdamme. Schließlich haben sich drittens einige Autoren dem schwierigen Terrain der Reichsfinanzverwaltung angenähert und das von den Reichsständen getragene System der Finanzierung einer Reichsarmee (»Römermonate«) bzw. die letztlich gescheiterten Versuche zur Einführung einer allgemeinen Reichssteuer (»Gemeiner Pfennig«) genauer erforscht. ${ }^{47}$

20 Von einigen neueren Arbeiten gestellt wird schließlich auch eine lange Zeit völlig vernachlässigte Frage: die nach einer Reichsgeschichte »von unten«, d. h. nach der Wahrnehmung und Praxis des Reichs auf der Ebene der sogenannten »einfachen« 
Untertanen. Als ein überaus fruchtbares Unterfangen hat sich dabei wiederum die Beschäftigung mit der Reichsgerichtsbarkeit erwiesen. Prozesse vor den höchsten Gerichten des Reiches zeigen nicht nur lokale Konflikte zwischen Untertanen und Obrigkeiten auf, sie lassen auch eine »kaiserliche Gerechtigkeit« sichtbar werden, die fester Bestandteil des bäuerlichen Rechtsverständnisses war. Das Reich fungierte hier also als eine wichtige, übergeordnete Instanz von Rechtsschutz und Rechtssicherheit ${ }^{48}$ - die Ergebnisse dieser Forschungen stützen somit die These von einer Verrechtlichung sozialer Konflikte im Alten Reich, die dazu beigetragen haben mochte, dass in den deutschen Territorien das Ancien Régime ohne revolutionäre Erschütterungen zu Ende ging. ${ }^{49}$ In Ansätzen erkennbar wird anhand solcher Prozesse auch, dass das Bewusstsein des Reiches besonders fest dort verankert war, wo territoriale Zersplitterung und multiple Zugehörigkeiten auf den Ebenen von Recht und Verwaltung herrschten (also vor allem im Südwesten, in Franken oder dem Rhein-Main-Gebiet). Das ist einerseits nicht überraschend, andererseits aber insofern bemerkenswert, als andere Befunde darauf hinweisen, dass das Reich und seine inneren wie äußeren Grenzen z. B. von Reisenden kaum wahrgenommen wurden und die räumliche Orientierung vielmehr anhand markanter Punkte (Städte) oder überschaubarer politischer Räume erfolgte. ${ }^{50}$ Beim Reisen mochte das Reich so nur mäßig präsent gewesen sein (wofür einen Grund die fehlenden Grenzkontrollen innerhalb wie außerhalb dargestellt haben dürften), in den Köpfen widerständiger Bauern oder um die Existenz ihrer Kleinstterritorien fürchtender Grafen und Herren hingegen war es dafür umso stärker gegenwärtig.

\section{Das Alte Reich und die französische Historiographie ${ }^{51}$}

21 Die Wahrnehmung des Reichs von »unten« und seine räumliche Konstruktion und Perzeption bilden zwei wichtige Themen, deren sich in den letzten Jahren ebenfalls französische Arbeiten angenommen haben. Ein drittes wichtiges Thema der französischen Forschung ist die konfessionelle Koexistenz im Reich. Diese französische »Reichsgeschichte« beschränkt sich im Wesentlichen auf eine überschaubare Gruppe von Historikerinnen und Historiker, die mehrheitlich dem Umfeld der 1977 von Robert Mandrou in Göttingen gegründeten Mission historique française en Allemagne (heute Institut français d'histoire en Allemagne in Frankfurt/M.) angehören; gleichzeitig kann sie jedoch an eine sehr fruchtbare deutsch-französische Zusammenarbeit etwa im Bereich der spätmittelalterlichen Politikgeschichte und Ritualforschung, aber auch der Historischen Anthropologie anschließen. ${ }^{52}$ Auf dem Gebiet der Frühen Neuzeit hingegen haben sich nur vereinzelt Forscher aus anderen Kontexten mit reichsgeschichtlichen Fragestellungen beschäftigt, vor allem der bereits erwähnte Jean-François Noël, der nicht selten Themen und Forschungsgegenstände aufgegriffen hat, noch bevor diese in der deutschen Geschichtswissenschaft die ihnen gebührende Aufmerksamkeit fanden. Dazu gehören die Reichsbelehnungen, die Reichsgrenzen und die Reichsgerichtsbarkeit. ${ }^{53}$ Neuland betreten hat er darüber hinaus auch mit einem Text zum Platz des Reiches im Bewusstsein des »einfachen Volkes« im 18. Jahrhundert, der in diese Ausgabe von Trivium aufgenommen wurde.

Stärker noch auf das Reich übertragen hat den Anspruch einer französischen Sozialgeschichte, die sich vor allem für Wahrnehmungsweisen und Handlungsstrategien der sozialen Akteure interessiert, Christophe Duhamelle mit 
seiner Studie zu den kollektiven dynastischen Strategien des rheinischen Stiftsadels im 17. und 18. Jahrhundert. ${ }^{54}$ Bereits diese Arbeiten füllen Leerstellen einer deutschen Reichsforschung, die ihren Blick lange Zeit vornehmlich auf institutionelle Strukturen und Verfassungsfragen gerichtet hatte. Besonders deutlich kamen die Anstöße durch die französische Historiographie aber bei einem anderen Aspekt: der Raumgeschichte. Trotz spatial turn und einer gesteigerten Aufmerksamkeit der deutschen Geschichtswissenschaft für die Geschichte der Grenzen wurden die maßgeblichen Arbeiten dazu bislang in Frankreich vorgelegt. Das gilt gleichermaßen für einen 2004 von Christine Lebeau herausgegebenen Sammelband, einen parallel dazu von Christophe Duhamelle koordinierten Themenschwerpunkt und einen 2010 von Hélène Miard-Delacroix, Béatrice von Hirschhausen und Guillaume Garner verantworteten, weiter ausgreifenden Band zur Raumgeschichte Deutschlands von der Frühen Neuzeit bis zur Gegenwart. ${ }^{55}$ In diesen Publikationen ist der Einfluss einer französischen Geschichtswissenschaft, die in Forschung und Lehre eng mit der Geographie verbunden ist, unverkennbar; beteiligt waren an ihnen allerdings immer auch deutsche Historikerinnen und Historiker. Gerade das Raumthema macht also die positiven Resultate einer deutsch-französischen Kooperation bei der Erforschung des Reichs deutlich, die auf anderen Gebieten bislang weitgehend noch in den Kinderschuhen steckt.

23 Entworfen wird in diesen Arbeiten - denen man noch Anne Saadas Studie zur Rezeption Diderots im deutschen "Kulturraum« des 18. Jahrhunderts sowie Axelle Chassagnettes Arbeit zum geographischen Wissen im 16. Jahrhundert hinzufügen $\mathrm{kann}^{56}$ - eine facettenreiche Geschichte des Raums des Alten Reichs, die über eingefahrene Analysekategorien wie die Territorien deutlich hinausgeht. Verhandelt wird in ihnen nicht der eine Raum des Reiches, sondern die vielfältigen, sich überlagernden und oft auch kreuzenden Vorstellungen und Praktiken, die dem Reich und seinen Gliedern eine sich im Zeitlauf immer wieder ändernde räumliche Gestalt gaben. Das Spektrum reicht dabei von den äußeren Reichsgrenzen und ihren Imaginationen über die verschiedenen Akteure (Reisende), Ebenen (Reichsstädte, Residenzstädte, Reichskreise, "Hauptstädte" des Reichs) und politischen bzw. ökonomischen Konstruktionen (Policey, Kameralismus, Zollgrenzen) des Raums bis hin $\mathrm{zu}$ den verschiedenen interpersonalen Netzwerken, die ihn oft quer zur Territorialstruktur durchzogen (Beziehungsnetze des Adels oder auch der Freimaurerei). Fassbar wird der Reichsraum hier also nicht als ein festgefügtes, "natürliches" Äußeres der Geschichte, sondern als eine dynamische und relationale Struktur, die auf einer aktiven menschlichen Handlungs- und Wahrnehmungspraxis beruhte.

24 Über die Perspektive des Raumes haben sich dem Reich auch einige Arbeiten der französischen Wirtschaftsgeschichte genähert. Dazu gehört eine Studie, die seinen Platz in den ökonomischen Diskursen im Deutschland der Aufklärung untersucht und dabei das Auseinanderklaffen zwischen den Kategorien des Kameralismus (insbesondere dem Begriff des Territoriums) und der konkreten räumlichen Gestalt des Reichs unterstrichen hat. ${ }^{57}$ Darüber hinaus war das Reich (wenn auch in verschieden starker Ausprägung) Gegenstand weiterer wirtschaftshistorischer Forschungen, etwa der Untersuchungen von Pierre Jeannin, der sich in mehreren Studien mit der frühneuzeitlichen Geld- und Handelsgeschichte in Deutschland auseinandergesetzt und dabei immer wieder den hohen Stellenwert des institutionellen und politischen Rahmen des Reichs und die Verflechtungen zwischen politischen und ökonomischen 
Akteuren herausgearbeitet hat. Dieser Ansatz ist in den letzten Jahren von MarieLouise Kaplan und Vincent Demont fortgeführt worden, wobei auch hier immer wieder die Raumdimension mit einbezogen wurde. ${ }^{58}$

Ein dritter Schwerpunkt der französischen Reichsforschung wird schließlich von Arbeiten zur konfessionellen Koexistenz gebildet. Gemeinsam mit deutschen Texten zum Thema erforderte er eigentlich eine eigene Ausgabe von Trivium, denn gerade am Konfessionalisierungsparadigma ließen sich besonders gut die oft nur impliziten Vorstrukturierungen des geschichtswissenschaftlichen Feldes aufzeigen, die die thematischen Schwerpunktsetzungen, chronologischen Muster und methodischen Zugriffe in den jeweiligen nationalen Historiographien bestimmen. Eingegangen wird auf diesen Bereich deshalb hier nur kurz: Im Gegensatz zur deutschen Konfessionalisierungsforschung zeichnen sich die französischen Beiträge vor allem durch eine sozialgeschichtliche Perspektive aus, die sich nicht einer bestimmten konfessionellen Richtung zurechnen lässt und deshalb transversalen Problemstellungen nachgeht, die in der deutschen Geschichtswissenschaft mit ihrer lange Zeit binären Ausrichtung im konfessionellen Abgrenzungskampf fehlen. So arbeiten nur wenige deutsche Historikerinnen und Historiker sowohl zur katholischen wie zur protestantischen Seite - französische Arbeiten hingegen nehmen nahezu durchgängig beide in den Blick. Ein anderer typischer Unterschied zur deutschen Forschung, die zwischen Kirchengeschichte und Religionsgeschichte oszilliert und dabei immer den Staat als Fixpunkt im Auge hat, ist daneben die Orientierung auf alltagsgeschichtliche Fragestellungen. Die Leitfrage lautet hier nicht, wie sich eine bestimmte Konfession im Zusammenspiel von Kirche, Staat und Gesellschaft hat durchsetzen können, sondern wie der konfessionelle Unterschied im Alltag gelebt und konstruiert wurde. Schließlich verfolgen französische Arbeiten oft eine Perspektive der langen Dauer, gehen also nicht davon aus, dass nach 1648 die konfessionellen Verhältnisse im Reich festgezurrt waren, weshalb sich Anstrengungen zu ihrer Erforschung in der nachfolgenden Zeit weitgehend erübrigen.

Paradigmatisch für die französische Forschung zur Geschichte der Konfessionalisierung im Reich stand zunächst vor allem Étienne François' Studie zum bikonfessionellen Augsburg. ${ }^{59}$ Dort wird nicht nur geschildert, wie Protestanten und Katholiken einen Stadtraum zwar gemeinsam bewohnten, gleichzeitig aber eigenen Abgrenzungsbestrebungen entsprechend so aneigneten, dass hier eine "unsichtbare Grenze« entstand, die mitunter von Haustür zu Haustür verlief. François nutzte den konfessionellen Unterschied auch als einen Einstieg, um sich mit generellen sozialhistorischen Fragen wie der demographischen Entwicklung, der Wohnsituation oder der Vornamensgebung auseinanderzusetzen. Seine Pionierstudie trägt in ihrer Verbindung aus historischer Demographie und Geschichte der Mentalitäten somit deutlich »französische" Züge und öffnete die Konfessionalisierungsforschung auch in Deutschland für eine ganze Reihe neuer Quellen, methodischer Ansätze und thematischer Fragestellungen. Zu nennen sind aber auch die Arbeiten von Patrice Veit, der das bislang weitgehend Spezialisten vorbehaltene evangelische Kirchenlied zum Ausgangspunkt einer Sozialgeschichte des Luthertums gemacht hat, oder die Arbeiten von Claire Gantet zur Sozialgeschichte des Westfälischen Friedens und zur konfessionellen Prägung des Träumens in der Frühen Neuzeit. ${ }^{60}$ Schließlich hat Christophe Duhamelle in den letzten Jahren mit seinen Forschungen zum katholischen Eichsfeld der französischen Konfessionalisierungsforschung - und darüber hinaus der französischen Reichsforschung überhaupt - neuen Schwung verliehen. Auch sein Fokus 
liegt auf der komplexen »Mischung von Nebeneinander, Miteinander und Gegeneinander" einer konfessionellen Koexistenz im Alltag, daneben aber auch auf anderen sozialhistorischen Fragestellungen wie beispielsweise der Geschichte von Grenzen oder von Herrschaft im Dorf. Dabei verweist er immer wieder auf die Bedeutung des Reichsrechts für die paritätische Ausgestaltung der konfessionellen Beziehungen im Reich, aber auch für das Verhältnis von Obrigkeit und Untertanen in einem von kleinteiligen Strukturen gekennzeichneten politischen Verband, der zahlreiche Chancen bot für eigenwillige Interpretationen, Umgehungen und Durchbrechungen von Macht. ${ }^{61}$

\section{Zur Auswahl der Texte dieser Ausgabe}

Die in diese Ausgabe von Trivium aufgenommenen Texte möchten eine Illustration sein für eine Reihe der hier angesprochenen Probleme und Tendenzen der Forschung. Ihre Auswahl hat keinen kanonischen Charakter - andere Herausgeber hätten sich möglicherweise für andere Texte entschieden. Ein wichtiges Kriterium war eine gewisse Repräsentativität der Beiträge für die Entwicklung der Reichsforschung in den letzten Jahrzehnten. Es ging uns also nicht allein um Aktualität, weshalb auch einige Artikel Eingang gefunden haben, deren Erstveröffentlichung bereits einige Zeit zurückliegt. Mit allen Texten verfolgen wir das Ziel, der Wissenschaft auch über den begrenzten Bereich der deutsch-französischen Forschungskooperation hinaus einige wesentliche Ergebnisse der Reichsgeschichte nahezubringen und damit auch Leserinnen und Leser zu erreichen, die sich bislang kaum oder gar nicht mit diesem Thema beschäftigt haben. Gleichwohl war uns nicht daran gelegen, eine Art »Lehrbuch" oder »Reader« zum Alten Reich vorzulegen. Unser Anliegen ist es vielmehr, die Besonderheiten der Reichsforschung diesseits und jenseits des Rheins herauszustellen (ohne dabei die bereits existierenden Verbindungen unter den Tisch fallen $\mathrm{zu}$ lassen) und so einen bescheidenen Beitrag dazu zu leisten, dass Wissenschaftlerinnen und Wissenschaftler aus beiden Historiographien in Zukunft ihre Forschungen stärker noch als bisher miteinander verknüpfen.

Eröffnet wird der Schwerpunkt mit zwei zentralen Texten einer Debatte, die die deutsche Geschichtswissenschaft Ende der 1990er Jahre beschäftigte, also zu einer Zeit, in der das wiedervereinigte Deutschland auf der Suche nach seiner zukünftigen Rolle in einem zusammenwachsenden Europa war. Wie bereits zur Zeit der »Reichseuphorie« in den 1970er und 80er Jahren war das Alte Reich nun erneut in den Fokus einer politischen Identität auf der Suche nach sich selbst geraten. Der Beitrag von Georg Schmidt benennt die begrifflichen Antipoden dieser Diskussion - „Sonderweg und Modell für Europa« oder "Staat der deutschen Nation« - bereits im Titel und nimmt damit die alte Auseinandersetzung um den Charakter des Reichs wieder auf, um ihr eine neue Wendung zu geben: Das Reich, so sein zentrales Argument, sei in der Form eines »komplementären Reichs-Staats« Staat und Nation der Deutschen in der Frühen Neuzeit gewesen. Das Attribut "komplementär« weist dabei darauf hin, dass die Staatlichkeit im Reich zunächst von den Territorien ausging, die Schmidt als besondere Staaten ansieht, die einer gemeinsamen höheren Gewalt unterworfen waren. Diese Fürstenstaaten und andere typische Formen einer begrenzten Staatlichkeit im Reich ergänzten sich $\mathrm{zu}$ »einer gemeinsamen, auf Deutschland gerichteten Handlungseinheit «, dem »komplementären Reichs-Staat « ${ }^{62}$. Die anderswo einheitliche 
Staatsgewalt war im Reich also auf verschiedene institutionelle und räumliche Ebenen verteilt, was auch bedeutete, dass der gesamtstaatliche Rahmen Voraussetzung für die Ausbildung von Staatlichkeit auf der territorialen Ebene war (und nicht ein Faktor ihrer Behinderung, wie dies die traditionelle Geschichtsschreibung behauptet hatte).

Verknüpft mit dieser neuen Interpretation war das Anliegen, den deutschen Fall aus seinem Status eines Solitärs im Gefüge des europäischen Staatensystems der Frühen Neuzeit herauszulösen. Mit der Figur der »komplementären Staatlichkeit«, so Schmidt, verliere die deutsche Geschichte ihre Besonderheit - und das Reich seine immer wieder bemühte Vorbildrolle für ein modernes, eben nicht nationalstaatlich geprägtes Europa gleich mit. Diese Absicht hatte eine geschichtspolitische Stoßrichtung: die Konstruktion einer deutschen Tradition, deren Gründungsmythos sich nicht auf den von außen angestoßenen Untergang (Napoleon) und die Rettung durch Preußen berief. Staat und Nation in Deutschland mussten nicht auf den Trümmern des Reichs wachsen, denn sie waren bereits vorher da. Auf den ersten Blick war diese Auffassung durchaus attraktiv, befreite sie die Diskussion doch von einer etwas angestaubten Selbstbescheidung, ohne in die preußische Machtstaatstheorie zurückzufallen (wobei Schmidt das Kunststück gelang, der bisherigen Historiographie eine unzulässige Vereinnahmung des Alten Reichs für aktuelle Orientierungsbedürfnisse vorzuwerfen und gleichzeitig selbst eine neue, auf ebensolche Legitimationsbedürfnisse zugeschnittene Meistererzählung anzubieten). In ihr spiegelten sich aber auch interne Konflikte der Geschichtswissenschaft - etwa der Kampf um Deutungshoheit zwischen der noch relativ jungen Subdisziplin »Frühe Neuzeit« und der etablierten »neueren Geschichte«, die mit dem Modernisierungsparadigma seit mehr als zwei Jahrzehnten den historiographischen Grundton vorgab. Die Verlagerung einer deutschen »Nation" in die Vormoderne muss deshalb auch als der Versuch gelesen werden, Deutungsmacht in einem Feld zu gewinnen, das bislang von Historikern des 19. und 20. Jahrhunderts dominiert wurde..$^{63}$ Hier nun blieb Schmidts Versuch weitgehend erfolglos - auch und besonders aufgrund des Widerstands der eigenen Kollegen.

Als Anwalt der Gegenseite trat in der Debatte Heinz Schilling auf, der bezeichnenderweise gleich zu Beginn seines Beitrags mitteilte, er sei eigentlich gar kein »Reichshistoriker" (was recht gut veranschaulicht, dass es in dieser Diskussion eben nicht nur um das Alte Reich ging). Die "feindliche Übernahme" der Reichsgeschichte durch die Kategorien und Interpretamente der neueren Geschichte wies er gleichwohl mit Verve und zahlreichen in den Fußnoten versteckten Tiefschlägen zurück. Seine Aufgabe war es, die Diskussion mit Verweis auf Volker Press (pikanterweise der Doktorvater Schmidts) und Bernd Roeck in alte, gemäßigtere Bahnen zurückzulenken. Sein Gegenvorschlag trug zwar eingestandenermaßen einen recht "monströsen Titel«, vermied aber alle teleologischer Tendenzen und Vereinnahmungen verdächtige Begrifflichkeiten wie "Staat" und »Nation«, um das Reich gleichermaßen in seinen modernen wie nicht-modernen Elementen zu erfassen. Im Deutungskampf trug er damit den Sieg davon. Denn der Rückgriff auf die Vorstellung eines "Systems«, das sich nur "teilweise« erneuerte und nur "partiell« zur Anpassung an eine Staatswerdung fähig war, wie sie sich zur gleichen Zeit auf der Ebene der Territorien bzw. in anderen europäischen Ländern vollzog, bedeutete den letztlich überzeugenderen Versuch, eine komplexe Terminologie zu finden, die den komplexen Funktionsmechanismen des Reichs Rechnung trug (und Schmidts Idee einer Komplementarität zumindest in Teilen integrierte). Nachhaltig prägen konnte Schilling die Reichsforschung damit jedoch ebenfalls nicht - zu sperrig war die begriffliche 
Konstruktion und zu stark zeichnete sich bereits um das Jahr $2000 \mathrm{ab}$, dass die wirklichen Neuerungen auf dem Gebiet der Reichsgeschichte ganz woanders erbracht werden sollten (siehe oben). Sein relativ lapidar am Beispiel einzelner Aspekte - von der Wehrverfassung bis zur konfessionellen Koexistenz, vom ökonomischen Handeln bis zum sakralen Charakter des Reichs - erbrachter Nachweis, dass das Reich eben doch kein Staat war, dürfte jedoch für die nächste Zukunft jeden Versuch, das Gegenteil zu behaupten, zu einem schwierigen bis unmöglichen Unterfangen gemacht haben. ${ }^{64}$

Die folgenden drei Texte widmen sich weniger grundsätzlichen, aber ebenso wichtigen Fragen für das Verständnis der Reichsgeschichte und weisen insbesondere auf neuere Ansätze hin, die eingeführte institutionen- oder verfassungsgeschichtliche Fragestellungen hinter sich lassen: Wolfgang Behringers Aufsatz widmet sich so der Geschichte der Reichspost und ihrer Rolle als Teil einer "Kommunikationsrevolution", die sich im Reich parallel zur »Medienrevolution« des Buchdrucks vollzogen hat. Ohne die »Kommunikationsinfrastruktur« der Post, so seine These, hätte der Reichstag seine Funktion als politisches Forum des Reichs im 16. Jahrhundert nicht erfüllen können. Darüber hinaus war sie wichtiges Element in den Herrschaftskonzeptionen des Kaisers, der sie auf den Weg gebracht hatte und unterhielt. Dabei zeigt sich einerseits, wie stark der Reichsraum in der Frühen Neuzeit mit gesamteuropäischen Kommunikationsnetzen verknüpft war (insbesondere nach Italien, woher ebenfalls die Familie der Reichspostmeister von Taxis stammte, aber auch in die Niederlande oder nach Spanien), und andererseits, wie stark seine territoriale Zersplitterung nicht etwa die Entwicklung der Post hemmte, sondern sie im Gegenteil nachgerade beförderte war die Post doch eines der wenigen Mittel, mit denen sich den Nachteilen ebendieser Zersplitterung begegnen ließ. Das war Voraussetzung für eine im europäischen Vergleich einzigartige Situation: Im Reich passte sich die geographische Verteilung der Institutionen (und insbesondere die des Reichstags) dem entstehenden Transportnetz der Post an, während es in anderen europäischen Ländern eher umgekehrt war.

Der Beitrag von Harriet Rudolph beschäftigt sich mit einem zentralen Akteur des Reichs, dem Kaiser. Dabei schließt sie an Ansätze einer Kulturgeschichte des Politischen an, die die lange Zeit von der Historiographie als inhaltsleere Maskerade verunglimpften zeremoniellen und rituellen Elemente der politischen Kommunikation im Reich in den Blick nehmen. ${ }^{65} \mathrm{Ihr}$ Vergleich der Kaiserkrönungen von 1562 (Maximilian II.) und 1790 (Leopold II.) und der damit verbundenen Feierlichkeiten unterstreicht zunächst das Gewicht dieser performativen Akte, die das Reich nicht allein symbolisch darstellten, sondern seine Existenz wesentlich begründeten. Diese elementaren politischen Funktionen von Königswahl und Kaiserkrönung erklären auch, warum sie - trotz einiger Veränderungen im Detail - bis zum Ende des Reichs nichts von ihrer Bedeutung (Herrscherlegitimation) und ihrer Faszination für die Zeitgenossen einbüßten. Das Krönungsfest charakterisiert Rudolph dabei als »Verfassungsfest« des Alten Reiches, in dem nicht nur die Reichsverfassung, sondern auch die Verfassungskultur der gastgebenden Reichsstadt und - über die anwesenden und teilnehmenden Reichsstände - der multipolare und multilokale Charakter der Reichsstruktur insgesamt zum Ausdruck gebracht wurden. In der Form einer mit der Zeit steigenden Teilhabe breiter sozialer Schichten transportierten die Feierlichkeiten darüber hinaus Partizipationsansprüche. Über konfessionelle und Standesunterschiede hinweg wurde durch die Krönungen und die sie begleitenden Feste so die Einheit des Reiches nicht nur abgebildet, sondern performativ immer wieder erst geschaffen. Die sinnstiftende Kraft solcher Zeremonien erschöpfte sich also nicht in ihrer rechtlichen 
Bestimmung, sondern resultierte vor allem aus der rituellen Herstellung einer sakral aufgeladenen Gemeinschaft, die Kaiser, Reichsstände und Reichsuntertanen über alle territorialen Grenzen hinweg miteinander verband.

Der bereits vor längerer Zeit erstmals erschienene Text von Heinz Duchhardt macht schließlich mit zwei charakteristischen, dennoch aber nur wenig bekannten Elementen der Reichsgeschichte vertraut: der Reichsritterschaft und der Reichsgerichtsbarkeit. Sein Beitrag zeichnet sich dadurch aus, dass er - als einer unter wenigen - einen sozialgeschichtlichen Blick auf das Reich wirft und konkret der Frage nachgeht, welche Informationen zum sozialen Gefüge sich aus dem Verhältnis von Ritterschaft und Reichsgerichtsbarkeit gewinnen lassen. Die reichsunmittelbaren Ritter waren eine mehrere Hundert Familien umfassende Gruppe freier Adliger vor allem in Franken, Schwaben und im Rheinland, die nur dem Kaiser als Lehnsherr unterstanden und nur ihm direkt Abgaben leisteten (in Form der sogenannten "Charitativsubsidien«). ${ }^{66} \mathrm{An}$ den Reichsinstitutionen (Reichstag, Reichskreise) partizipierten sie hingegen nur mittelbar oder gar nicht. Das galt zunächst auch für das Reichskammergericht, eines der beiden Reichsgerichte, dem die Reichsritter im 16. Jahrhundert noch grundsätzlich ablehnend gegenüberstanden, da sie sich durch seine Existenz in ihrem Selbstverständnis bedroht fühlten. Ab der Mitte des 17. Jahrhunderts hingegen änderte sich dies. Von nun erkannte die Ritterschaft in der Reichsgerichtsbarkeit nicht nur ein effizientes Instrument, um die eigene Sonderstellung im Reichsverband zu sichern, das Reichskammergericht wurde gleichzeitig auch zu einem wichtigen Betätigungsfeld, da aufgrund von Reformation und Säkularisierungen nicht mehr genügend Positionen in den Stiftskapiteln zu besetzen waren. Im 18. Jahrhundert war das Gericht schließlich zu einer nachgerade ritterschaftlich dominierten Institution geworden. Über die Details dieses Wandels hinweg gibt Duchhardts Text so Einblick in einige wichtige Aspekte einer Sozialgeschichte des Alten Reiches, die geprägt war von einem konfliktreichen Miteinander von Großen und Kleinen (hier den Reichsrittern mit ihren oft nur winzigen Territorien, die permanent von der Mediatisierung durch ihre mächtigeren Nachbarn bedroht waren), von einer ständigen Konkurrenz zwischen Angehörigen unterschiedlicher Standesgruppen und von den Dynamiken einer sozialen Schichtung, die offenbar erstaunlich durchlässig war. Schließlich macht sein Artikel noch einmal die für die Reichsverfassung elementare Rolle persönlicher Abhängigkeitsverhältnisse deutlich (nur die enge Bindung an den Kaiser erlaubte es den Reichsrittern, von einer Reichsinstitution zu profitieren, zu deren Unterhalt sie nicht den geringsten Beitrag leisteten).

Eine sozial- bzw. mentalitätsgeschichtliche Perspektive nimmt auch der Beitrag von Jean-François Noël ein, der die Reihe der aus dem Französischen übersetzen Texte einleitet und einer an sich simplen Frage nachgeht: Was wussten im 18. Jahrhundert die sogenannten »einfachen Leute" vom Reich und welchen Platz hatte dieses in ihren politischen, rechtlichen und sozialen Vorstellungen? Die bisherige Forschung hat sich immer wieder mit dem »Reichspatriotismus« auseinandergesetzt - also jenen Bekenntnissen zum Reich, die man in gelehrten oder literarischen Abhandlungen finden kann. ${ }^{67}$ Die Ebene der Normalsterblichen « hingegen blieb ausgeblendet, auch aufgrund vermeintlich fehlender Quellen, aus denen heraus sich ein solches »Reichsbewusstsein« der Untertanen hätte rekonstruieren lassen. Noëls Beitrag nun zeigt auf, welche Materialien für eine solche Untersuchung zur Verfügung stehen können: die weltliche und geistliche Architektur, Symbole (z. B. auf Münzen), Kirchengebete mit Widmungen an den Kaiser, Lieder und Reime, Katechismen und 
Schulbücher, aber auch die großen öffentlichen Zeremonien und Rituale des Reiches wie die Kaiserkrönungen, an denen ebenfalls das Volk teilhatte. In allen diesen Quellen lassen sich jeweils verschiedene Spuren finden, die auf das populäre Bild des Reiches schließen lassen. Vor allem aber weist der Text auf eine Quellengattung hin, die inzwischen auch von der Forschung zur Reichsgerichtsbarkeit und ihrer Nutzung durch die Untertanen intensiv rezipiert wurde: Eingaben, Klagen und Suppliken an den Reichshofrat bzw. an den Kaiser höchstselbst. Dort artikulierte sich nicht nur eine oft erstaunliche Kenntnis der vielschichtigen institutionellen Realität des Reiches, sondern auch eine Fähigkeit, diese Vielfalt der Ebenen im Interesse eigener Vorstellungen von Ordnung oder Herrschaft zu nutzen. Selbst wenn einige Briefe ein an Naivität grenzendes Vertrauen in die kaiserlichen Schutzfähigkeiten zeigen oder nur die sprichwörtliche "Bauernschläue» findiger Untertanen zu bestätigen scheinen, lassen sie in der Gesamtschau doch den Kaiser als eine wichtige Instanz der Gerechtigkeit und des Rechts erscheinen, die auch im Bewusstsein von Bewohnern scheinbar »reichsferner« Gegenden fest verankert war. Schließlich zeigt der Text gleichsam en passant auf, wie stark Informationen etwa zur Reform der Strafgesetze im Reich zirkulierten und auch die unteren Bevölkerungsschichten erreichten.

Mit Étienne François' Beitrag zur Hierarchie der frühneuzeitlichen Städte im Alten Reich folgt erneut ein Text, der bereits vor längerer Zeit erschienen ist - eine gleichrangige Publikation jüngeren Datums liegt jedoch nicht vor. ${ }^{68}$ Anliegen des Artikels ist ein vergleichender sozialgeschichtlicher Blick auf die Städtelandschaft des Alten Reichs um 1600 und 1800. Einander gegenüber gestellt werden die Entwicklung und der funktionale Dualismus zwischen den traditionellen Wirtschaftszentren (Handels- und Hansestädte sowie freie Städte) und den nach 1650 aufstrebenden Hauptbzw. Residenzstädten in den Territorien, von denen sie in ihrer Bedeutung schließlich weitgehend verdrängt werden sollten. Dieses spezifische städtische Wachstum und die mit ihm verbundene demographische Dynamik verfolgt François nicht nur anhand von Bevölkerungszahlen und -zusammensetzung, sondern auch an anderen Kriterien, etwa der Stadtplanung und der konkreten Gestaltung des städtischen Raums oder der Verbindung zwischen Städtelandschaft und künstlerischer Produktion. Damit schildert er nicht nur eine Umkehrung in der städtischen Hierarchie des frühneuzeitlichen Deutschlands, sondern legt auch den Grundstein für eine Raumgeschichte des Alten Reichs, wie sie in der deutschen Historiographie bis heute kaum oder gar nicht praktiziert wird. Wenig überraschend zeigt die methodische Herangehensweise des Autors im Vergleich zur deutschen Forschung deshalb auch einige Eigenheiten: Dazu gehört zuerst die Verwendung von Karten nicht zu illustrativen Zwecken, sondern als ein Mittel der Erkenntnisgewinnung (für viele französische Historiker ist es immer wieder frappierend zu sehen, wie selten ihre deutschen Kollegen zu Karten greifen, auch wenn sie zu raumgeschichtlichen Fragestellungen arbeiten). Auch steht der Text unverkennbar in der Tradition einer französischen Stadtgeschichtsschreibung mit ihrer auf der Grundlage von seriellen Daten aufbauenden Verbindung von Kartographie und Demographie. Mit diesem methodischen Instrumentarium gelingt es François, einige charakteristische Züge der Städtelandschaft des Alten Reichs herauszuarbeiten, die sich als ein multipolarer Raum aus mehreren, sich überlagernden Städtenetzen ohne eindeutiges Gravitätszentrum kennzeichnen lässt, dessen konkrete Struktur einem permanentem historischen Wandel unterlag. schließlich der Artikel von Christophe Duhamelle. In seiner - ebenfalls von Karten 
gestützten - Argumentation steht ein Phänomen im Mittelpunkt, das lange Zeit zu den bevorzugten "Hassobjekten « einer Historiographie gehörte, die in der "Staatswerdung" der Territorien den Königsweg der historischen Entwicklung gesehen hatte: die Exklaven (also jene zahllosen Bestandteile von Territorien, die abgetrennt vom Hauptteil im Land verstreut lagen). Dieses typische Element der politischen Geographie des Reichsraums nimmt Duhamelle zum Ausgangspunkt für eine Analyse der politischen und territorialen Zersplitterung des Reichs und der konkreten Praktiken, mit denen die sozialen Akteure ihr begegneten. Dabei wird zunächst noch einmal der politische Charakter des Reichs als Lehnsverband deutlich, dessen Strukturprinzip nicht die geographische Kontinuität, sondern die Sammlung von Herrschaftsrechten war. Einen eingehenden Blick wirft der Text darüber hinaus auf die Allgegenwart und die Porosität der Grenzen, die mit dieser besonderen Situation verbunden waren. Sie verliehen dem Raum des Reiches nicht nur eine fraktale Gestalt, die - wie das auch hier zitierte Beispiel der Reichspost zeigt - keinesfalls Mobilität behindern musste. Daneben waren sie auch ein wichtiger Ort, an dem Zugehörigkeiten und (z. B. konfessionelle) Identitäten verhandelt wurden. Schließlich erwies sich an ihnen noch einmal das Reichsrecht als wichtige Instanz der Konfliktlösung (oder besser: der Kanalisierung von Konflikten) und als Klammer, die trotz der Zersplitterung das Ganze zusammenhielt. Auch aufgrund dieser juristischen Rahmung konnten die Exklaven somit zu einem strukturierenden Element des Reichsraums werden: Hier wurden (selbst im preußischen Fall) komplexe Herrschaftssituationen ausgehandelt und Handlungsspielräume erweitert, die diese komplexen Herrschaftssituationen aneigneten und ausnutzten. Dabei lässt sich im Handeln der Akteure noch einmal eine subtile Kenntnis der verschiedenen räumlich-politischen Referenzebenen ablesen, die jede Reduzierung der geschichtlichen Entwicklung im frühneuzeitlichen Deutschland auf die eine politische Wirklichkeit der »Territorialstaaten« wirkungsvoll dementiert. Mit anderen Worten: Das Spiel mit den Maßstabsebenen, das viele Historiker heute erst mühsam erlernen müssen, war im Alltag der Bewohner des Reichs eine offenbar selbstverständliche Angelegenheit.

In der deutsch-französischen Gesamtschau zeigen die ausgewählten Texte so eines deutlich: Die Reichsgeschichte hat sich in den letzten Jahren von einer relativ geschlossenen Subdisziplin zu einer Forschungsrichtung entwickelt, die zunehmend offen ist für neue sozial-, kultur- und raumgeschichtliche Fragestellungen. Stärker noch als bisher kann sie jedoch in Zukunft von der produktiven Spannung profitieren, die unterschiedliche disziplinäre Traditionen oder verschiedene chronologische Zuschnitte in den beiden nationalen Historiographien bedeuten. Erste Anfänge für eine verstärkte Zusammenarbeit zwischen der deutschen und der französischen Forschung zum Alten Reich sind gemacht - und mit der vorliegenden Ausgabe von Trivium verbinden wir die Hoffnung, sie möge nicht nur der Ausgangspunkt für eine intensivere Wahrnehmung der im jeweils anderen Land erzielten Forschungsergebnisse, sondern auch für weitere solcher Kooperationen sein. 


\section{BIBLIOGRAPHIE}

Ackermann, J. (2002): Verschuldung, Reichsdebitverwaltung, Mediatisierung. Eine Studie zu den Finanzproblemen der mindermächtigen Stände im Alten Reich: Das Beispiel der Grafschaft YsenburgBüdingen, 1687-1806, Marburg: Hessisches Landesamt für geschichtliche Landeskunde.

Amend-Traut, A. (2009): Wechselverbindlichkeiten vor dem Reichskammergericht. Praktiziertes Zivilrecht in der Frühen Neuzeit, Köln / Weimar / Wien: Böhlau.

Aretin, K. O. von (1991): »Reichspatriotismus«, in: Birtsch, G. (Hg): Patriotismus = Interdisziplinäre Halbjahresschrift zur Erforschung des 18. Jahrhunderts und seiner Wirkungsgeschichte, 4 (2), S. 25-36.

Aretin, K. O. von (1993): Das Alte Reich, Bd. 1: Föderalistische oder hierarchische Ordnung (1648-1684), Stuttgart: Klett-Cotta.

Aretin, K. O. von (1997a): Das Alte Reich, Bd. 2: Kaisertradition und österreichische Großmachtpolitik (1684-1745), Stuttgart: Klett-Cotta.

Aretin, K. O. von (1997b): Das Alte Reich, Bd. 3: Das Reich und der österreichisch-preußische Dualismus (1745-1806), Stuttgart: Klett-Cotta.

Arndt, J. (1991): »Die Grafschaft Lippe und die Institutionen des Heiligen Römischen Reiches im 17. und 18. Jahrhundert«, Zeitschrift für historische Forschung, 18, S. 149-176.

Babel, R. (2005): Deutschland und Frankreich im Zeichen der habsburgischen Universalmonarchie. 15001650, Darmstadt: WBG (Deutsch-französische Geschichte, 3).

Bähr, M. (2012): Die Sprache der Zeugen. Argumentationsstrategien bäuerlicher Gemeinden vor dem Reichskammergericht (1693-1806), Konstanz: UVK.

Baumann, A. (2001): Die Gesellschaft der frühen Neuzeit im Spiegel der Reichskammergerichtsprozesse. Eine sozialgeschichtliche Untersuchung zum 17. und 18. Jahrhundert, Köln / Weimar / Wien: Böhlau.

Behringer, W. (2003): Im Zeichen des Merkur. Reichspost und Kommunikationsrevolution in der Frühen Neuzeit, Göttingen: Vandenhoeck \& Ruprecht.

Blaich, F. (1970): Die Wirtschaftspolitik des Reichstags im Heiligen Römischen Reich, Stuttgart: G.Fischer.

Blaschke, K. (Hg.) (2007): Moritz von Sachsen. Ein Fürst der Reformationszeit zwischen Territorium und Reich, Leipzig: Sächsische Akademie der Wissenschaften.

Blickle, P. (2003): Von der Leibeigenschaft zu den Menschenrechten. Eine Geschichte der Freiheit in Deutschland, München: C.H. Beck.

Blickle, P. (2008): Das alte Europa. Vom Hochmittelalter bis zur Moderne, München: C.H. Beck.

Bog, I. (1959): Der Reichsmerkantilismus. Studien zur Wirtschaftspolitik des Heiligen Römischen Reiches im 17. und 18. Jahrhundert, Stuttgart: G. Fischer.

Braubach, M. u. a. (1974): Vom Westfälischen Frieden bis zur Französischen Revolution (= Gebhardt Handbuch der deutschen Geschichte, Bd.10), 9., neu bearb. Aufl., hg. von H. Grundmann, München: dtv.

Braun, G. (2008): Von der politischen zur kulturellen Hegemonie Frankreichs. 1648-1789, Darmstadt: WBG (Deutsch-französische Geschichte, 4). 
Braun, G. (2010): La connaissance du Saint-Empire en France du baroque aux Lumières (1643-1756), München: Oldenbourg.

Bretschneider, F. (Hg.) (2011): Praxis der Grenze - Konflikte und Umgänge mit Territorialgrenzen im Alten Reich, Stuttgart (= Jahrbuch für Regionalgeschichte, 29).

Burgdorf, W. (1998): Reichskonstitution und Nation: Verfassungsreformprojekte für das Heilige Römische Reich Deutscher Nation im politischen Schriftum von 1648 bis 1806, Mainz: Zabern.

Burkhardt, J. (2002): »Europäischer Nachzügler oder institutioneller Vorreiter? Plädoyer für einen neuen Entwicklungsdiskurs zur konstruktiven Doppelstaatlichkeit des frühmodernen Reichs«, in: Schnettger, M. (Hg.): Imperium Romanum - Irregulare Corpus - Teutscher Reichs-Staat: das Alte Reich im Verständnis der Zeitgenossen und der Historiographie, Mainz: Zabern, S. 297-316.

Burkhardt, J. (2006): Vollendung und Neuorientierung des frühmodernen Reiches, 1648-1763 (= Gebhardt - Handbuch der deutschen Geschichte, Bd. 11), 10. Aufl., hg. von A.Haverkamp, W. Reinhard, J. Kocka, W. Benz, Stuttgart: Klett-Cotta.

Chaline, O. (1998): La Reconquête catholique de l'Europe centrale (XVIe-XVIIIe siècle), Paris: Cerf.

Chassagnette, A. (im Druck): Mesurer et décrire. Savoir géographique et cartographie dans l'espace germanique protestant (des années 1530 aux années 1620), Genf: Droz.

Christmann, T. (1988): Das Bemühen von Kaiser und Reich um die Vereinheitlichung des Münzwesens: zugleich ein Beitrag zum Rechtsetzungsverfahren im Heiligen Römischen Reich nach dem Westfälischen Frieden, Berlin: Duncker \& Humblot.

Demel, W. (2005): Reich, Reformen, sozialer Wandel 1763-1806(= Gebhardt - Handbuch der deutschen Geschichte, Bd. 12), 10. Aufl., hg. von A.Haverkamp, W. Reinhard, J. Kocka, W. Benz, Stuttgart: Klett-Cotta.

Demont, V. (2008): L'orfêvrerie-joaillerie à Francfort-sur-le-Main et à Hambourg (1585-1648): marché et acteurs, Ms., unveröff. Dissertation, Universität Paris 7.

Diestelkamp, B. (1999): Recht und Gericht im Heiligen Römischen Reich, Frankfurt/M.: Klostermann.

Dotzauer, W. (1998): Die deutschen Reichskreise (1383-1806). Geschichte und Aktenedition, Stuttgart: Steiner.

Duchhardt, H. (1991): Deutsche Verfassungsgeschichte 1495-1806, Stuttgart: Kohlhammer.

Duhamelle, C. (1998a): »Das Alte Reich im toten Winkel der französischen Historiographie«, in: Schnettger, M. (Hg.): Imperium Romanum - irregulare corpus - Teutscher Reichs-Staat: das Alte Reich im Verständnis der Zeitgenossen und der Historiographie, Mainz: Zabern, S. 207-219.

Duhamelle, C. (1998b): L'héritage collectif. La noblesse d'Église rhénane, 17e-18e siècles. Paris: Éditions de l'EHESS.

Duhamelle, C. (1998c): „La noblesse du Saint-Empire et le crédit: autour du >Von den Reichsständischen Schuldenwesen`de Johann Jacob Moser (1774)«, European University Institute Working Papers, HEC, 98 (2), S. 1-15.

Duhamelle, C. (Hg.) (2004): Les espaces du Saint-Empire à l'époque moderne = Histoire, Économie \& Société, 23 (1), Paris: Armand Colin.

Duhamelle, C. (2007): »Territoriale Grenze, konfessionelle Differenz und soziale Abgrenzung. Das Eichsfeld im 17. und 18. Jahrhundert«, in: François, E. / Seifarth, J. / Struck, B. (Hg.): Die Grenze als Raum, Erfahrung und Konstruktion. Deutschland, Frankreich und Polen vom 17. bis zum 20. Jahrhundert, Frankfurt/M.: Campus, S. 33-51. 
Duhamelle, C. (2010): La frontière au village. Une identité catholique allemande au temps des Lumières, Paris: Éditions de la MSH.

Eichhorn, J. (2006): Geschichtswissenschaft zwischen Tradition und Innovation. Diskurse, Institutionen und Machtstrukturen der bundesdeutschen Frühneuzeitforschung, Göttingen: V\&R unipress.

Erbe, M. (2007): Frühe Neuzeit, Stuttgart: Kohlhammer (Grundkurs Geschichte).

François, E. (1991): Die unsichtbare Grenze. Protestanten und Katholiken in Augsburg, Sigmaringen: Thorbecke; frz. Ausgabe: Protestants et catholiques en Allemagne. Identités et pluralisme. Augsbourg, 1648-1806, Paris: Albin Michel, 1993.

Friedrich, S. (2007): Drehscheibe Regensburg. Das Informations- und Kommunikationssystem des Immerwährenden Reichstags um 1700, Berlin: Akademie Verlag.

Fürnrohr, W. (1963): Der immerwährende Reichstag zu Regensburg: Das Parlament des alten Reiches. Zur 300-Jahrfeier seiner Eröffnung 1663, Regensburg / Kallmünz: Lassleben.

Gabel, H. (1995): Widerstand und Kooperation. Studien zur politischen Kultur rheinischer und maasländischer Kleinterritorien (1648-1794), Tübingen: Bibliotheca academica.

Gantet, C. (2001): La paix de Westphalie (1648). Une histoire sociale, XVIIe-XVIIIe siècle, Paris: Belin.

Gantet, C. (2010): „Le rêve dans l'Allemagne du XVIe siècle. Appropriations médicales et recouvrements confessionnels«, Annales. Histoire, sciences sociales, 65 (1), S. 39-62.

Garner, G. (2005): État, économie et territoire en Allemagne. L'espace dans le caméralisme et l'économie politique (1740-1820), Paris: Éditions de l'EHESS.

Gestrich, A. (1994): Absolutismus und Öffentlichkeit. Politische Kommunikation in Deutschland zu Beginn des 18. Jahrhunderts, Göttingen: Vandenhoeck \& Ruprecht.

Göttmann, F. (1991): Getreidemarkt am Bodensee: Raum, Wirtschaft, Politik, Gesellschaft (1650-1810), St. Katharinen: Scripta Mercaturae Verlag.

Gotthard, A. (1992): Säulen des Reiches: die Kurfürsten im frühneuzeitlichen Reichsverband, 2 Bde., Husum: Matthiesen.

Gotthard, A. (2003a): Das Alte Reich 1495-1806, Darmstadt: WBG (Geschichte kompakt).

Gotthard, A. (2003b): „Vormoderne Lebensräume. Annäherungsversuch an die Heimaten des frühneuzeitlichen Mitteleuropäers«, Historische Zeitschrift, 276, S. 37-73.

Gotthard, A. (2007): In der Ferne. Die Wahrnehmung des Raums in der Vormoderne, Frankfurt/M. / New York: Campus.

Hartmann, P. C. (1991): »Messefreiheiten, Messeprivilegien, Messerecht und Fremdenrecht in der frühen Neuzeit«, in: Koch, R. / Pohl, H. (Hg.): Brücke zwischen den Völkern - Zur Geschichte der Frankfurter Messe, Bd. 1, Frankfurt/M.: Historisches Museum Frankfurt, S. 249-261.

Hartmann, P. C. (Hg.) (1997): Der Mainzer Kurfürst als Reichserzkanzler: Funktionen, Aktivitäten, Ansprüche und Bedeutung des zweiten Mannes im alten Reich, Stuttgart: Steiner.

Hartmann, P. C. (Hg.) (2002): Die Mainzer Kurfürsten des Hauses Schönborn als Reichserzkanzler und Landesherren, Frankfurt/M.: Peter Lang.

Hartung, F. (1914): Deutsche Verfassungsgeschichte, Leipzig / Berlin: Teubner, Neuauflagen 1959, 1964 und 1969.

Heil, D. (Hg.) (1998): Die Reichspolitik Bayerns unter der Regierung Herzog Albrechts V. (1550-1579), Göttingen: Vandenhoeck \& Ruprecht. 
Herborn, W. (1991): „Die mittelalterlichen Messen im deutschsprachigen Raum«, in: Koch, R. / Pohl, H. (Hg.): Brücke zwischen den Völkern - Zur Geschichte der Frankfurter Messe, Bd. 1, Frankfurt/ M.: Historisches Museum Frankfurt, S. 67-84.

Histoire / Geschichte, Bd. 1: Europa und die Welt von der Antike bis 1815/L'Europe et le monde de l'Antiquité à 1815. Stuttgart (Klett) und Paris (Nathan) 2008 und 2011.

Iggers, G. G. u. a. (Hg.) (1998): Die DDR-Geschichtswissenschaft als Forschungsproblem, München: Oldenbourg (= Historische Zeitschrift, Beiheft N.F., 27).

Jäger, F. (Hg.) (2005-2012): Enzyklopädie der Neuzeit, 16 Bde., Stuttgart: Metzler.

Jeannin, P. (1996): Marchands du Nord. Espaces et trafics à l'époque moderne, Paris: Éditions rue d'Ulm.

Jeannin, P. (2001): Change, crédit et circulation monétaire à Augsbourg au milieu du 16e siècle, Paris: Armand Colin.

Jeannin, P. (2002): Marchands d'Europe. Pratiques et savoirs à l'époque moderne, Paris: Éditions rue d'Ulm.

Kaufmann, G. H. (1889): »Die Universitätsprivilegien der Kaiser«, Deutsche Zeitschrift für Geschichtswissenschaft, 1, S. 118-165.

Lanzinner, M. (1993): Friedenssicherung und politische Einheit des Reiches unter Kaiser Maximilian II. (1564-1576), Göttingen: Vandenhoeck \& Ruprecht.

Lanzinner, M. / Schormann, G. (2008): Konfessionelles Zeitalter 1555-1618. Dreißigjähriger Krieg 16181648(= Gebhardt - Handbuch der deutschen Geschichte, Bd. 10), 10. Aufl., hg. von A.Haverkamp, W. Reinhard, J. Kocka, W. Benz, Stuttgart: Klett-Cotta.

Lebeau, C. (1996): Aristocrates et grands commis à la Cour de Vienne (1748-1791). Le modèle français, Paris: CNRS Éditions.

Lebeau, C. (Hg.) (2004): L'espace du Saint-Empire du Moyen Âge à l'époque moderne, Straßburg: Presses universitaires de Strasbourg.

Miard-Delacroix, H. / Hirschhausen, B. von / Garner, G. (Hg.) (2010): Espaces de pouvoir, espaces d'autonomie en Allemagne, Villeneuve d'Ascq: Presses du Septentrion.

Mohnhaupt, H. (im Druck): »Bestrebungen zur Vereinheitlichung der Privilegienvielfalt im Bereich des Handwerks im Kurfürstentum Brandenburg (1734-1736) «, in: Garner, G. (Hg.): L'économie du privilège en Europe occidentale (XVIe-XIXe siècle), Frankfurt/M.: Klostermann.

Monnet, P. (2004): „Courriers et messages: un réseau urbain de communication dans les pays d'Empire à la fin du Moyen Âge«, in: Boudreau, C. / Gauvard, C. / Hebert, M. / Fianu, K. (Hg.): Information et société en Occident à la fin du Moyen Age, Paris: Publications de la Sorbonne, S. 281306.

Moraw, P. (1989): »Hoftag und Reichstag von den Anfängen im Mittelalter bis 1806«, in: Schneider, H.-P. / Zeh, W. (Hg.): Parlamentsrecht und Parlamentspraxis in der Bundesrepublik Deutschland. Ein Handbuch, Berlin / New York: de Gruyter, S. 3-47.

Neuhaus, H. (1997): Das Reich in der Frühen Neuzeit, München: Oldenbourg (= Enzyklopädie deutscher Geschichte, 42).

Neuhaus, H. (1999): »Die Begründung der Leipziger Messe und das Heilige Römische Reich Deutscher Nation in den Jahren 1497/1507«, in: Zwahr, H. / Topfstedt, T. / Bentele, G. (Hg.): 
Leipzigs Messen 1497-1997. Gestaltwandel - Umbrüche - Neubeginn, 1. Teilbd.: 1497-1914, Köln /

Weimar / Wien: Böhlau, S. 51-60.

Noël, J.-F. (1966): »Les problèmes de frontières entre la France et l'Empire dans la seconde moitié du XVIIIe siècle«, Revue historique, 90 (235), S. 333-346.

Noël, J.-F. (1968): »Zur Geschichte der Reichsbelehnungen im 18. Jahrhundert«, Mitteilungen des Österreichischen Staatsarchivs, 21, S. 106-122.

Noël, J.-F. (1970): »Der Reichshofrat und das Verfassungsleben der Reichsstädte zur Zeit Josephs II.«, Esslinger Studien. Jahrbuch für Geschichte der oberdeutschen Reichsstädte,16, S. 121-131.

Noël, J.-F. (1976): Le Saint-Empire, Paris: PUF (Reihe »Que sais-je?«, 1646), überarbeitete Neuauflagen 1986 und 1993.

Noël, J.-F. (1977): »Les pays rhéno-moséllans et la Chambre impériale de justice

(Reichskammergericht). 1500-1600«, in: Théorie et pratique politiques à la Renaissance (Actes du XVIIe colloque international d'études humanistes), Paris: Vrin, S. 159-187.

Ortlieb, E. (2001): Die kaiserlichen Kommissionen des Reichshofrats und die Regelung von Konflikten im Alten Reich (1637-1657), Köln / Weimar / Wien: Böhlau.

Pelus-Kaplan, M.-L. (2009): »Migrations, mobilité et culture d'entreprise dans les villes de la Hanse au XVIe siècle: l'exemple des entreprises lubeckoises«, in: Maitte, C. / Mande, I. / Martini, M. / Terrier, D. (Hg.): Entreprises en mouvement. Migrants, pratiques entrepreneuriales et diversités culturelles dans le monde (XVe-XXe siècles), Valenciennes: Presses Universitaires de Valenciennes, S. 47-62.

Peters, J. (1991): »Eigensinn und Widerstand im Alltag «, Jahrbuch für Wirtschaftsgeschichte, 2, S. 85103.

Petry, D. (2011): Konfliktbewältigung als Medienereignis. Reichsstadt und Reichshofrat in der Frühen Neuzeit, Berlin: Akademie Verlag.

Press, V. (1997a):Das Alte Reich. Ausgewählte Aufsätze, hg. von J. Kunisch, Berlin:Duncker \& Humblot.

Press, V. (1997b): »Das römisch-deutsche Reich - ein politisches System in verfassungs- und sozialgeschichtlicher Fragestellung«, in: ders.: Das Alte Reich. Ausgewählte Aufsätze, hg. von J. Kunisch, Berlin:Duncker \& Humblot, S. 18-41.

Press, V. (1998): Adel im alten Reich. Gesammelte Vorträge und Aufsätze, hg. von F. Brendle und A. Schindling in Verbindung mit M. Rudersdorf und G. Schmidt, Tübingen: Bibliotheca academica.

Ranft, A. u. a. (Hg.) (2003): Traditionen - Visionen. 44. Deutscher Historikertag in Halle an der Saale 2002, München: Oldenbourg.

Rauscher, P. / Edelmayer, F. / Lanzinner, M. (Hg.) (2003): Finanzen und Herrschaft. Materielle Grundlagen fürstlicher Politik in den habsburgischen Ländern und im Heiligen Römischen Reich im 16. Jahrhundert, Wien / München: Oldenbourg.

Rauscher, P. u. a. (2004): Zwischen Ständen und Gläubigern. Die kaiserlichen Finanzen unter Ferdinand I. und Maximilian II. (1556-1576), Wien / München: Oldenbourg.

Reinhard, W. (2001): Probleme deutscher Geschichte 1495-1806. Reichsreform und Reformation 14951555(= Gebhardt - Handbuch der deutschen Geschichte, Bd. 9), 10. Aufl., hg. von A.Haverkamp, W. Reinhard, J. Kocka, W. Benz, Stuttgart: Klett-Cotta. 
Riedenhauer, E. (Hg.) (1994): Landeshoheit. Beiträge zur Entstehung, Ausformung und Typologie eines Verfassungselements des Römisch-deutschen Reiches, München: Kommission für Bayerische Landesgeschichte (= Studien zur bayerischen Verfassungs- und Sozialgeschichte, 16).

Rudolph, H. (2011): Das Reich als Ereignis. Formen und Funktionen der Herrschaftsinszenierung bei Kaisereinzügen (1558-1618), Köln / Weimar / Wien: Böhlau.

Saada, A. (2003): Inventer Diderot: les constructions d'un auteur en Allemagne au XVIIIe siècle, Paris: CNRS Éditions.

Sailer, R. (1999): Untertanenprozesse vor dem Reichskammergericht. Rechtsschutz gegen die Obrigkeit in der zweiten Hälfte des 18. Jahrhunderts, Köln / Weimar / Wien: Böhlau.

Sandl, M. (1999): Ökonomie des Raumes. Der kameralwissenschaftliche Entwurf der Staatswirtschaft im 18. Jahrhundert, Köln / Weimar / Wien: Böhlau.

Scheutz, M. (2012): »>Mental Maps« von Vagierenden in der Frühen Neuzeit«, Volkskunde in Sachsen, 24, S. 111-140.

Schilling, H. (2001): »Reichs-Staat und frühneuzeitliche Nation der Deutschen oder teilmodernisiertes Reichssystem - Überlegungen zu Charakter und Aktualität des Reiches«, Historische Zeitschrift, 272, S. 377-395.

Schindling, A. (1991): Die Anfänge des Immerwährenden Reichstags zu Regensburg. Ständevertretung und Staatskunst nach dem Westfälischen Frieden, Mainz: Zabern.

Schmidt, G. (1999): Geschichte des Alten Reiches. Staat und Nation in der Frühen Neuzeit 1495-1806, München: C.H. Beck.

Schmidt, G. (2001): »Das frühneuzeitliche Reich - komplementärer Staat und föderative Nation«, Historische Zeitschrift, 273, S. 371-399.

Schmidt, G. (2009): Wandel durch Vernunft. Deutsche Geschichte im 18. Jahrhundert, München: C.H. Beck.

Schmidt, R. (1999): »Päpstliche und kaiserliche Universitätsprivilegien im späten Mittelalter«, in: Dölemeyer, B. / Mohnhaupt, H. (Hg.): Das Privileg im europäischen Vergleich, Bd. 2, Frankfurt/M.: Klostermann, S. 143-154.

Schmitt, J.-C. / Oexle, O. G. (Hg.) (2003): Les tendances actuelles de l'histoire du Moyen Âge en France et en Allemagne, Paris: Publications de la Sorbonne.

Schneider, K. (1995): Die Münz- und Währungspolitik des Oberrheinischen Reichskreises im 18. Jahrhundert, Koblenz: Numismatischer Verlag Forneck.

Schnettger, M. (Hg.) (2002): Imperium Romanum - Irregulare Corpus - Teutscher Reichs-Staat: das Alte Reich im Verständnis der Zeitgenossen und der Historiographie, Mainz: Zabern.

Schnettger, M.(2004): „Le Saint-Empire et ses périphéries: l'exemple de l'Italie«, Histoire, économie et société, 23 (1), S. 7-23.

Schnettger, M.(2006): »Principe sovrano« oder »civitas imperialis«? Die Republik Genua und das Alte Reich in der Frühen Neuzeit (1556-1797), Mainz: Zabern.

Schnettger, M. (2007): »Von der `Kleinstaaterei` zum `komplementären Reichs-Staat «. Die Reichsverfassungsgeschichtsschreibung seit dem Zweiten Weltkrieg«, in: Kraus, H.-C. / Nicklas, T. (Hg.): Geschichte der Politik. Alte und neue Wege. München: Oldenbourg (= Historische Zeitschrift, Beihefte N.F., 44), S. 129-154. 
Schorn-Schütte, L. (1984): »Territorialgeschichte - Provinzialgeschichte - Landesgeschichte Regionalgeschichte. Ein Beitrag zur Wissenschaftsgeschichte der Landesgeschichtsschreibung«, in: Jäger, H. u. a. (Hg.): Civitatis Communitas. Studien zum europäischen Städtewesen, Köln / Wien: Böhlau, S. 390-416.

Schulze, W. (1978): »Die Erträge der Reichssteuern zwischen 1576 und 1606«, Jahrbuch für die Geschichte Mittel- und Ostdeutschlands, 27, S. 169-185.

Schulze, W. (1980): Bäuerlicher Widerstand und feudale Herrschaft in der frühen Neuzeit, Stuttgart / Bad Cannstatt: Frommann-Holzboog.

Stollberg-Rilinger, B. (1999): Vormünder des Volkes? Konzepte landständischer Repräsentation in der Spätphase des Alten Reiches, Berlin: Duncker \& Humblot.

Stollberg-Rilinger, B. (2004): "Symbolische Kommunikation in der Vormoderne. Begriffe Forschungsperspektiven - Thesen «, Zeitschrift für historische Forschung, 31, 2004, S. 489-527; franz. Übers.: »La communication symbolique à l'époque pré-moderne. Concepts, thèses, perspectives de recherche«, Trivium, 2-2008 (http://trivium.revues.org/1152; Stand 30.5.2013).

Stollberg-Rilinger, B. (2006): Das Heilige Römische Reich Deutscher Nation. Vom Ende des Mittelalters bis 1806, München: C.H. Beck.

Stollberg-Rilinger, B. (2008): Des Kaisers alte Kleider. Verfassungsgeschichte und Symbolsprache des Alten Reichs, München: C.H. Beck; franz.: Les vieux habits de l'empereur. Une histoire culturelle des institutions du Saint-Empire à l'époque moderne, übers. v. C. Duhamelle, Paris: Éditions de la MSH, 2013.

Stollberg-Rilinger, B. (2009): »Le rituel de l'investiture dans le Saint-Empire de l'époque moderne: histoire institutionnelle et pratiques symboliques«, Revue d'histoire moderne et contemporaine, 56 (2), S. 7-29.

Stolleis, M. (1991): »Reichspublizistik und Reichspatriotismus vom 16. zum 18. Jahrhundert«, in: Birtsch, G. (Hg): Patriotismus = Interdisziplinäre Halbjahresschrift zur Erforschung des 18. Jahrhunderts und seiner Wirkungsgeschichte, 4 (2), S. 7-23.

Treitschke, H. von (1879): Deutsche Geschichte im neunzehnten Jahrhundert, Bd. 1: Bis zum zweiten Pariser Frieden. Leipzig: Hirzel.

Ullmann, S. (2002): »Landesherr und Kaiser im Spiegel eines Zeugenverhörs des Reichshofrats aus den Jahren 1575-1579«, in: Fuchs, R.-P. / Schulze, W. (Hg.): Wahrheit, Wissen, Erinnerung.

Zeugenverhörprotokolle als Quelle für soziale Wissensbestände der Frühen Neuzeit, Münster u. a.: LIT Verlag, S. 257-290.

Veit, P. (2002): »Pratiques de piété, chant et musique. À propos de la culture luthérienne au XVIIe siècle«, in: ders. / Valentin, J.-M. (Hg.): La confessionnalisation dans le Saint-Empire, XVIeXVIIIe siècles = Études Germaniques, 57 (3), S. 473-487.

Völker-Rasor, V. (Hg.) (2010): Frühe Neuzeit, Neuauflage (erstmals 2000), München: Oldenbourg (Oldenbourg Geschichte-Lehrbuch).

Vogler, G. (1983): Die Gewalt soll gegeben werden dem gemeinen Volk. Der deutsche Bauernkrieg 1525, Berlin: Dietz.

Weber, E. E. (1992): Städtische Herrschaft und bäuerliche Untertanen in Alltag und Konflikt. Die Reichsstadt Rottweil und ihre Landschaft vom 30jährigen Krieg bis zur Mediatisierung, 2 Bde., Rottweil: Stadtarchiv Rottweil.

Wendehorst, S. / Westphal, S. (Hg) (2006): Lesebuch Altes Reich, München: Oldenbourg. 
Westphal, S. (2002): Kaiserliche Rechtsprechung und herrschaftliche Stabilisierung. Reichsgerichtsbarkeit in den thüringischen Territorialstaaten, 1648-1806, Köln / Weimar / Wien: Böhlau.

Whaley, J. (2011): Germany and the Holy Roman Empire, Bd. 1: Maximilian I to the Peace of Westphalia, 1493-1648, Bd. 2: The Peace of Westphalia to the Dissolution of the Reich, 1648-1806, Oxford: Oxford University Press.

Willoweit, D. (1975): Rechtsgrundlagen der Territorialgewalt. Landesobrigkeit, Herrschaftsrechte und Territorium in der Rechtswissenschaft der Neuzeit, Köln / Wien: Böhlau.

Wolfram, H. (Hg.) (1994-1995): Österreichische Geschichte, 10 Bde., Wien: Ueberreuther.

Wrede, M. (2007): »Entre Empereur, Empire et nation: l'essor de ^l'esprit politique en Allemagne moderne (XVIIe-XVIIIe siècles)«, Revue historique, 643 (3), S. 623-652.

Wüst, W. (Hg.) (2000): Reichskreis und Territorium: Die Herrschaft über der Herrschaft? Supraterritoriale Tendenzen in Politik, Kultur, Wirtschaft und Gesellschaft. Ein Vergleich süddeutscher Reichskreise, Stuttgart: Thorbecke.

Wunder, B. (1996): »Der Kaiser, die Reichskreise und der Chausseebau im 18. Jahrhundert«, Zeitschrift für Neuere Rechtsgeschichte, 18 (1/2), S. 1-22.

\section{Quellen}

Die peinliche Gerichtsordnung Kaiser Karls V. und des Heiligen Römischen Reichs von 1532 (Carolina), hg. u. erläutert von F.-C. Schroeder, Stuttgart: Reclam, 2000.

Die Reichspolizeiordnungen von 1530, 1548 und 1577. Historische Einführung und Edition, hg. von M. Weber, M. Frankfurt/M.: Klostermann, 2002.

\section{NOTES}

1. $\mathrm{Zu}$ solchen Initiativen gehören etwa die vom Deutschen Historischen Institut (DHI) Paris betreute Deutsch-französische Geschichte in 11 Bänden, insbesondere Bd. 3 (Babel [2005]) und Bd. 4 (Braun [2008]) sowie das deutsch-französische Schulbuch Histoire / Geschichte (2008 und 2011).

2. Whaley (2011).

3. Noël (1976).

*. NDLR: ein solches einsprachiges Format wurde z. B. für die 5. Trivium-Ausgabe zur »Soziologie der Konventionen« gewählt.

4. Vgl. dazu auch das Projekt »Histoire du Saint-Empire - regards croisés franco-allemands«, eine Kooperation der École des hautes études en sciences sociales (Centre de recherches historiques und Centre Georg Simmel), der École Normale Supérieure de Lyon sowie der Universitäten Münster und Mainz mit Unterstützung des Centre interdisciplinaire d'études et de recherches sur l'Allemagne (nähere Informationen unter http://www.saint-empire.fr) sowie die Sektion "Die Tradition einer multinationalen Reichsgeschichte heute» auf dem Historikertag 2002 - eine Zusammenfassung im Berichtsband: Ranft u. a. (2003), S. 70-77.

5. Vgl. Eichhorn (2006), zur Reichsgeschichte bes. S. 311-392. Aus Sicht der Reichsgeschichte selbst Press (1997b) sowie Schnettger (2007).

6. Gebhardt - Handbuch der deutschen Geschichte: Braubach u. a. (1974); Burkhardt (2006).

7. Vgl. beispielhaft Hartung (1914).

8. Die DDR-Geschichtswissenschaft folgte zunächst der mit einer Abwertung Preußens einhergehenden neuen Sicht auf das Reich, wandte sich dann aber im Zuge der »Erbe«- 
Diskussion und der Konstruktion einer eigenen »sozialistischen Nation« vom Reich wieder ab. Vgl. Iggers u. a. (1998).

9. Als grundlegende Überblicksdarstellungen, welche die Ergebnisse dieser Forschungen zusammenfassen, lassen sich neben dem bereits erwähnten 11. Bd. die Bde. 9, 10 und 12 des Gebhardt nennen: Reinhard (2001); Lanzinner / Schormann (2008); Demel (2005). Daneben auch die Darstellungen von Aretin (1993), (1997a), (1997b) und Press (1997a). Als Einführung in die Forschung auch Duchhardt (1991) und Neuhaus (1997). Zudem sei auf die einschlägigen Artikel zum Reich in: Jäger (2005-2012) verwiesen, die neben dem Sachstand häufig auch eine Einführung in die derzeitigen Forschungsdebatten bieten. Karl Otmar von Aretin, von 1968 bis 1994 Direktor des Mainzer Instituts für Europäische Geschichte, und Volker Press, von 1980 bis zu seinem plötzlichen Tod 1993 Professor in Tübingen, bildeten mit ihren jeweiligen Schülerinnen und Schülern die maßgeblichen Pole der Reichsgeschichte in der Bundesrepublik.

10. Fürnrohr (1963). Solche Versuche einer historischen Legitimation der bundesrepublikanischen Gegenwart durch das Alte Reich dauern teilweise bis heute an. Vgl. etwa Burkhardt (2002).

11. Bog (1959) und Blaich (1970).

12. Stollberg-Rilinger (1999), bes. S. 2-5.

13. Schnettger (2002). Zur Außenwahrnehmung des Reichs vgl. etwa Wrede (2007) und Braun (2010).

14. Gotthard (1992).

15. Vgl. einführend Stollberg-Rilinger (2006), Gotthard (2003a) sowie Wendehorst / Westphal (2006).

16. Diese Veränderungen fanden Niederschlag auch in den Raumkonzeptionen politischer Macht. Im Laufe der Frühen Neuzeit verband sich die traditionelle Landeshoheit, d. h. die Herrschaftsgewalt eines Reichsstandes in seinem Territorium, immer mehr mit der Idee einer politisch-geographischen Geschlossenheit dieses Territoriums unter Ausschaltung intermediärer Gewalten. Vgl. Riedenhauer (1994).

17. Im Gegensatz zu älteren Darstellungen hat die Forschung inzwischen herausgearbeitet, dass die Reichsverfassung seit dem Westfälischen Frieden von einer ständigen Diskussion über ihre Reform begleitet wurde, die Niederschlag in einer breiten publizistischen Reflexion gefunden hat. Vgl. vor allem Burgdorf (1998).

18. Die Erforschung der Territorialgrenzen im Reich steht - trotz reichlich vorhandenen Quellenmaterials - erst am Anfang. Vgl. Bretschneider (2011), S. 35-110 sowie Gotthard (2003b).

19. Treitschke (1879), S. 7.

20. Schmidt, G. (1999).

21. Eichhorn (2006). Die aktuelle Gleichzeitigkeit von »Reichsferne« und »Reichsnähe« der deutschen Frühneuzeitforschung lässt sich exemplarisch an den folgenden zwei Einführungen ablesen: Völker-Rasor (2010 [2000]) - hier kommt das Reich in keiner einzigen Kapitelüberschrift vor - und Erbe (2007) - hier steht das Reich gleichberechtigt neben Spanien oder Frankreich. Beide Publikationen verstehen sich gleichermaßen als eine Annäherung an die Frühe Neuzeit aus europäischer bzw. Welt-Perspektive.

22. Dabei spielt eine Rolle, dass die frühneuzeitliche Kriminalgesetzgebung maßgeblich auf der territorialen Ebene stattfand. Ausnahme war nur die als Rahmengesetz fungierende Carolina. Vgl. Die peinliche Gerichtsordnung Kaiser Karls V. (2000).

23. Die Reichspolizeiordnungen von 1530, 1548 und 1577 (2002). Vgl. auch das am Max-Planck-Institut für Europäische Rechtsgeschichte unter der Leitung von Karl Härter und Michael Stolleis durchgeführte Projekt eines »Repertoriums der Policeyordnungen« (http://www.rg.mpg.de/de/ forschung/policeyordnungen), das Reich und Territorien einbezieht.

24. Sandl (1999), bes. S. 37-45.

25. Schmidt, G. (1999). Gleiches gilt auch für Schmidt, G. (2009). 
26. $\mathrm{Zu}$ Reichsitalien vgl. etwa Schnettger (2006) und (2004).

27. Vgl. Wolfram (1994-1995).

28. Koordiniert wird die Edition von der Historischen Kommission bei der Bayerischen Akademie der Wissenschaften (http://www.historischekommission-muenchen.de/index.php? seite=projekte). Zum Reichstag vgl. einführend Moraw (1989); Schindling (1991).

29. Bähr (2012). Zu den Reichsgerichten insgesamt vgl. einführend den Themenschwerpunkt »Reichsgerichtsbarkeit«, in: zeitenblicke 3 (2004), Nr. 3 (http://www.zeitenblicke.de/2004/03/ index.htm) sowie Diestelkamp (1999).

30. Dotzauer (1998) und Wüst (2000).

31. Wichtige Quellen, insbesondere die Akten des Mainzer Reichserzkanzleramts, liegen so heute im Haus-, Hof- und Staatsarchiv in Wien.

32. Behringer (2003). Kommunikationsnetze durchzogen das Reich jedoch schon seit dem Spätmittelalter, vgl. etwa Monnet (2004).

33. Herborn (1991); Hartmann (1991); Neuhaus (1999).

34. Schmidt, R. (1999) sowie immer noch Kaufmann (1889).

35. Eine Rolle spielt dabei die spezifische Ausrichtung der deutschen Landesgeschichte, die bis heute über weite Strecken Züge einer Legitimationswissenschaft der regionalen Identitätsbildung trägt. Einer Beschäftigung mit dem Reich war und ist das eher hinderlich. Vgl. Schorn-Schütte (1984).

36. Vgl. exemplarisch Heil (1998) oder Blaschke (2007).

37. Hartmann (1997) und (2002).

38. Arndt (1991). Zu Thüringen Westphal (2002).

39. Petry (2011); Ackermann (2002); Ortlieb (2001); Duhamelle (1998c).

40. Diese Perspektive wurzelt auch in der Tradition des Begriffs des »Territoriums«. Dieses galt bereits der frühneuzeitlichen Rechtswissenschaft als der weitgehend autonome Herrschaftsbereich eines Reichsstandes. Vgl. Willoweit (1975).

41. Stollberg-Rilinger (2008). Die französische Übersetzung dieses für die aktuelle deutsche Reichsforschung zentralen Buches erscheint nahezu zeitgleich mit dieser Ausgabe von Trivium in der »Bibliothèque allemande« der Éditions de la Maison des sciences de l'homme unter dem Titel: Les vieux habits de l'empereur. Une histoire culturelle des institutions du Saint-Empire à l'époque moderne. Vgl. auch Stollberg-Rilinger (2004) und (2009).

42. Friedrich (2007). Vgl. auch Gestrich (1994).

43. Baumann (2001); Amend-Traut (2009).

44. Die Studie von Christmann (1988) unterstreicht, dass sich derartige Bestrebungen nicht auf die Münzordnungen des 16. Jahrhunderts beschränkten, sondern sich bis zum Ende des 18. Jahrhunderts fortsetzten.

45. Mohnhaupt (im Druck).

46. Wunder (1996); Göttmann (1991); Schneider (1995).

47. Vgl. neben den Vorarbeiten von Schulze (1978) und Lanzinner (1993) jetzt vor allem die Forschungen von Rauscher u. a. (2004) und Rauscher / Edelmayer / Lanzinner (2003).

48. Sailer (1999) und Ullmann (2002). Daneben die landesgeschichtlichen Fallstudien von Gabel (1995) und Weber (1992).

49. Vgl. Blickle (2008) sowie (2003). Blickles Sicht ist nicht unwidersprochen geblieben, weil sie der Tendenz zur Romantisierung eines vermeintlich friedfertigen und konfliktfreien Zusammenlebens in "Alteuropa« nur selten widersteht. Sie schließt jedoch an eine breite Forschung zum bäuerlichen Widerstand an, für die prominent die Arbeiten Winfried Schulzes stehen. Vgl. etwa Schulze (1980). Beigetragen haben zu ihr auch DDR-Historiker, vor allem Vogler (1983) und Peters (1991).

50. Gotthard (2007), bes. S. 105-107. Zu ähnlichen Ergebnissen kommt auch Scheutz (2012). 
51. Vgl. auch Duhamelle (1998a). Dort auch Hinweise zur älteren Reichsgeschichte in Frankreich. Nicht näher eingegangen werden kann hier auf die französische Tradition einer Geschichte der Habsburger, die sich bis heute fortsetzt. Vgl. etwa Chaline (1998) und (1996). Sie steht durchaus in thematischer Nähe zur Reichsgeschichte, beharrt aber gleichzeitig auf eigenen Akzentsetzungen, die das Reich bisweilen hinter dem - über Jahrhunderte von den Habsburgern gestellten - Kaiser zurücktreten lassen.

52. Vgl. insbesondere Schmitt / Oexle (2003).

53. Noël (1968), (1966), (1970) und (1977).

54. Duhamelle (1998b).

55. Lebeau (2004); Duhamelle (2004); Miard-Delacroix / Hirschhausen / Garner (2010).

56. Saada (2003); Chassagnette (im Druck).

57. Garner (2005).

58. Jeannin (2001), (2002), (1996); Pelus-Kaplan (2009); Demont (2008).

59. François (1991).

60. Veit (2002); Gantet (2001), (2010).

61. Duhamelle (2010); (2007), Zitat S. 46.

62. Das Zitat aus Schmidt, G. (1999), S. 44, wo das Konzept ausführlich erläutert und empirisch unterfüttert wird.

63. Ähnlich lassen sich auch andere Versuche interpretieren, vgl. etwa Burgdorf (1998).

64. Schilling stützte seine Argumentation auf eine eingehende Besprechung des Buches von Georg Schmidt in der Historischen Zeitschrift, der ältesten deutschen geschichtswissenschaftlichen Zeitschrift, die immer noch Gewicht im Feld hat. Vgl. Schilling (2001). Die Antwort von Georg Schmidt: vgl. Schmidt, G. (2001). Um den Rahmen der Debatte auf der Mainzer Tagung von 2001 $\mathrm{zu}$ erhalten, die auch und besonders aufgrund dieser Auseinandersetzung einen wichtigen Moment in der neueren Reichsforschung darstellte, haben sich die Herausgeber entschieden, die dort von Schmidt und Schilling vorgetragenen Texte in diese Ausgabe von Trivium aufzunehmen. Die Artikel beider Autoren in der Historischen Zeitschrift gehören jedoch in den gleichen, sich ergänzenden Debattenzusammenhang.

65. Dazu ausführlich Rudolph (2011). Vgl. auch die oben bereits ausführlich erwähnten Arbeiten von Barbara Stollberg-Rilinger.

66. Vgl. dazu auch Press (1998).

67. Burgdorf (1998); Stolleis (1991); Aretin (1991).

68. Der Umstand, dass François' Text bis heute nicht ins Deutsche übersetzt worden ist, zählt zu den Merkwürdigkeiten einer deutsch-französischen Zusammenarbeit in den Geschichtswissenschaften, zu deren maßgeblichen Akteuren der Autor selbst gehörte und gehört.

\section{INDEX}

Schlüsselwörter : Saint-Empire

Mots-clés : Altes Reich 


\section{AUTEURS}

\section{FALK BRETSCHNEIDER}

Falk Bretschneider ist Assistenzprofessor an der École des hautes études en sciences sociales (EHESS), Paris. Nähere Informationen finden Sie hier.

\section{GUILLAUME GARNER}

Guillaume Garner ist Dozent an der École Normale Supérieure (ENS) in Lyon. Nähere Informationen finden Sie hier. 